\title{
Null Killing vectors and geometry of null strings in Einstein spaces
}

\author{
Adam Chudecki
}

Received: 17 September 2013 / Accepted: 16 March 2014 / Published online: 31 March 2014 C The Author(s) 2014. This article is published with open access at Springerlink.com

\begin{abstract}
Einstein complex spacetimes admitting null Killing or null homothetic Killing vectors are studied. Such vectors define totally null and geodesic 2-surfaces called the null strings or twistor surfaces. Geometric properties of these null strings are discussed. It is shown, that spaces considered are hyperheavenly spaces $(\mathcal{H} \mathcal{H}$-spaces $)$ or, if one of the parts of the Weyl tensor vanishes, heavenly spaces ( $\mathcal{H}$-spaces). The explicit complex metrics admitting null Killing vectors are found. Some Lorentzian and ultrahyperbolic slices of these metrics are discussed.
\end{abstract}

Keywords Hyperheavenly spaces · Heavenly spaces · Isometric Killing vectors · Homothetic Killing vectors · Null Killing vectors · Null strings

\section{Introduction}

The idea of using the complex numbers in analysis of the spacetime is almost so old, as theory of relativity. [Einstein himself used the imaginary time coordinate in special theory of relativity]. However, advanced complex methods in general theory of relativity have been discovered in the sixties. R. Penrose [1] and Newman [2] introduced the spinorial formalism, Debney et al. [3] and others developed the null tetrad formalism. Several important works were dedicated to the methods of finding new metrics of real spacetime from already known metrics by appropriate complex transformations [4,5]. These methods had their origins in the work [6] where searching for the solutions to the Maxwell equations were considered. Then Newman [7] showed that each asymptotically flat spacetime defines some 4-dimensional complex analytic

\footnotetext{
A. Chudecki $(\varangle)$

Center of Mathematics and Physics, Technical University of Lodz, Al. Politechniki 11,

90-924 Lodz, Poland

e-mail: adam.chudecki@p.lodz.pl
} 
differential manifold endowed with a holomorphic Riemannian metric. This metric satisfies the vacuum complex Einstein equations and the self-dual or anti-self-dual part of its conformal curvature tensor (Weyl tensor) vanishes. Such a space was called by Newman the heavenly space $(\mathcal{H}$-space) .

Plebański [8] showed that vacuum complex Einstein equations for a heavenly space can be reduced to a single second order nonlinear partial differential equation, heavenly equation ( $\mathcal{H}$-equation), for one holomorphic function. Besides very interesting mathematical properties, the solutions of heavenly equation have been considered as "basic bricks" which could be used to construct the solutions of Einstein equations by appropriate superpositions of two such solutions [9]. This approach, however, appeared to be limited. This is why the new methods of looking for the real solutions from the complex ones have been developed. One of them is the hyperheavenly space theory.

Hyperheavenly spaces $(\mathcal{H} \mathcal{H}$-spaces) was introduced in 1976 in famous work by Plebański and Robinson [10] as a natural generalization of the heavenly spaces. Hyperheavenly spaces with cosmological constant $\Lambda$ are complex spacetimes with algebraically degenerate self-dual or anti-self-dual part of the Weyl tensor satisfying the vacuum Einstein equations with cosmological constant. The transparent advantage of hyperheavenly spaces theory is the reduction of Einstein equations to one, nonlinear differential equation of the second order, i.e. hyperheavenly equation.

Moreover, it has been pointed out that all the real, algebraically special, vacuum, Lorentzian metrics are hidden inside the hyperheavenly metrics! It seemed, that finding new real vacuum solutions of Einstein field equations with the Lorentzian signature was only a matter of time. It was enough to solve the hyperheavenly equation and then to find Lorentzian slices of respective complex spacetimes. This research programme, often called the Plebański programme has its origin in the works by Trautman [6] and Newman et al. [4,5] (see also [11]). Unfortunately, obtaining the real Lorentzian slices appeared to be more difficult then anyone suspected.

In order to better understand the problem, the structure of hyperheavenly spaces together with their spinorial description have been investigated by Plebański, Finley III et al's. [12-14]. Believing that symmetry of the spacetime simplifies the problem, some authors have studied Killing symmetries in heavenly and hyperheavenly spaces [15-18]. But Lorentzian slices still remain elusive. Except some examples [14,19-22] and discussions [23] no general techniques to find the Lorentzian (physical) slices have been presented. Exceptionally, some reality conditions have been analyzed [24], but practical applications of these ideas appeared to be problematic. Probably it was the reason why hyperheavenly machinery became less popular in nineties.

Within five last years hyperheavenly spaces found their place in deep mathematical considerations. Their relation to Walker and Osserman geometry has been noticed in 2008. Some transparent results have been obtained with help of hyperheavenly formalism $[25,26]$. It appeared, that $\mathcal{H} \mathcal{H}$-spaces are the most natural tool in investigating real spaces of the neutral (ultrahyperbolic) signature $(++--)$. Moreover, a few works devoted to Killing symmetries in heavenly and hyperheavenly spaces appeared [27-30]. These papers generalized the previous ideas of Plebański, Finley III and Sonnleitner [16-18]. Between Killing vectors especially useful are these ones, which are tangent to self-dual null strings. The existence of such (null) Killing vectors simplifies the hyperheavenly equation, making it solvable in majority of cases. 
The main aim of our work is to find all complex hyperheavenly and heavenly metrics admitting null homothetic and isometric Killing symmetry. Such metrics appear to be important in (++- -) real geometries. However, the existence of a null Killing vector appeared to be helpfull for finding the Lorentzian slices [28]. We develop this idea and examine all possible Lorentzian slices of the complex spacetimes admitting the null Killing vector.

It is well known [23] that if a complex spacetime admits any real Lorentzian slice then both self-dual and anti-self-dual part of the Weyl tensor must be of the same Petrov-Penrose type. So if this complex spacetime is a hyperheavenly space (with or without $\Lambda$ ) then by the Goldberg-Sachs theorem it admits both self-dual and antiself-dual congruences of null strings (i.e. totally null and geodesic 2-surfaces) which intersect each other and these intersections constitute the congruence of null geodesics. To assume the existence of null Killing vector field and identify this field with congruence of null geodesics seem to be the natural first steps in investigating Lorentzian slices.

The paper is organized as follows.

In Sect. 2 we investigate the general properties of Killing vectors, especially of null ones. Some useful theorems are given and relation between null Killing vector and null strings is pointed out. Then the detailed discussion on the possible Petrov-Penrose types admitting null Killing symmetry is presented. Section 3 is a concise summary of the properties of hyperheavenly spaces. The main goal of our work is to present explicit form of the metrics with null Killing symmetry. The results are gathered in Sects. 4 and 5. There are seven different hyperheavenly metrics with null isometric or homothetic Killing vector and five different heavenly metrics.

In (the most important) Sect. 6 we discuss the possible real slices of the metrics found in preceding sections. In order to find the Lorentzian slices we use the idea of complex coordinate transformations. We introduce the appropriate complex transformations of the complex metrics and then we replace all the holomorphic functions and coordinates by the real ones. After these steps we obtain, quite surprisingly, metrics with the Lorentzian signature. Our technique leads to a new method for obtaining the Lorentzian metrics. This method is different from the analysis of the reality conditions [24], superposition of two heavenly metrics [9] or complex transformation of the real metrics [6]. Finally Lorentzian slices of the type [II] and [D] are found. Then we discuss some metrics of the neutral signature. Two-sided Walker and globally Osserman spaces are obtained. The concluding remarks end our paper.

\section{Null Killing vectors and null strings}

2.1 Killing equations and their integrability conditions in spinorial formalism.

Let $\mathcal{M}$ be a 4-dimensional complex analytic differentiable manifold endowed with a holomorphic metric $d s^{2}$. Thus $\left(\mathcal{M}, d s^{2}\right)$ is a 4-dimensional holomorphic Riemannian manifold and one deals with complex relativity. The metric $d s^{2}$ on $\mathcal{M}$ can be written in terms of a null complex tetrad $\left(e^{1}, e^{2}, e^{3}, e^{4}\right)$ (where $e^{i}, i=1, \ldots, 4$ are co-vectors) 


$$
d s^{2}=2 e^{1} \underset{s}{\otimes} e^{2}+2 e^{3} \underset{S}{\otimes} e^{4}
$$

Let $\left(\partial_{1}, \partial_{2}, \partial_{3}, \partial_{4}\right)$ be the inverse basis of (complex) vectors. For our purposes it is useful to use the spinorial formalism. Thus we introduce the respective spinorial images of basis of co-vectors and vectors

$$
\left(g^{A \dot{B}}\right):=\sqrt{2}\left[\begin{array}{cc}
e^{4} & e^{2} \\
e^{1} & -e^{3}
\end{array}\right], \quad\left(\partial_{A \dot{B}}\right):=-\sqrt{2}\left[\begin{array}{cc}
\partial_{4} & \partial_{2} \\
\partial_{1} & -\partial_{3}
\end{array}\right], \quad A=1,2, \quad \dot{B}=\dot{1}, \dot{2}
$$

We use the following rules of manipulating the spinorial indices

$$
m_{A}=\epsilon_{A B} m^{B}, \quad m^{A}=m_{B} \epsilon^{B A}, \quad m_{\dot{A}}=\epsilon_{\dot{A} \dot{B}} m^{\dot{B}}, \quad m^{\dot{A}}=m_{\dot{B}} \epsilon^{\dot{B} \dot{A}}
$$

where $\epsilon_{A B}$ and $\epsilon_{\dot{A} \dot{B}}$ are the spinor Levi-Civita symbols

$$
\begin{array}{r}
\left(\in_{A B}\right):=\left[\begin{array}{cc}
0 & 1 \\
-1 & 0
\end{array}\right]=:\left(\in^{A B}\right), \quad\left(\epsilon_{\dot{A} \dot{B}}\right):=\left[\begin{array}{cc}
0 & 1 \\
-1 & 0
\end{array}\right]=:\left(\epsilon^{\dot{A} \dot{B}}\right) \\
\epsilon_{A C} \in^{A B}=\delta_{C}^{B}, \quad \epsilon_{\dot{A} \dot{C}} \in^{\dot{A} \dot{B}}=\delta_{\dot{C}}^{\dot{B}}, \quad\left(\delta_{C}^{A}\right)=\left(\delta_{\dot{C}}^{\dot{B}}\right)=\left[\begin{array}{cc}
1 & 0 \\
0 & 1
\end{array}\right]
\end{array}
$$

Correspondence between the null tetrad formalism and spinorial formalism is realized with the use of the spin-tensor $g^{a A \dot{B}}$ which is defined by the relation $g^{A \dot{B}}=g_{a}{ }^{A \dot{B}} e^{a}$. It is easy to see that $-\frac{1}{2} g^{a A \dot{B}} g_{b A \dot{B}}=\delta_{b}^{a}$ and $-\frac{1}{2} g^{a A \dot{B}} g_{a C \dot{D}}=\delta_{C}^{A} \delta_{\dot{D}} \dot{B}$. The operators $\partial^{A \dot{B}}$ and $\nabla^{A \dot{B}}$ are the spinorial images of operators $\partial^{a}$ and $\nabla^{a}$, respectively, given by

$$
\partial^{A \dot{B}}=g_{a}^{A \dot{B}} \partial^{a} \quad \nabla^{A \dot{B}}=g_{a}^{A \dot{B}} \nabla^{a}
$$

In spinorial notation the metric can be written in the form

$$
d s^{2}=-\frac{1}{2} g_{A \dot{B}} \underset{s}{\otimes} g^{A \dot{B}}
$$

[In complex relativity the 1 -forms $g^{A \dot{B}}$ are unrelated. In real relativity there are some constraints for the 1 -forms $g^{A \dot{B}}$. For example, for the real spaces of the signature $(++--)$ there must be $\overline{g^{A \dot{B}}}=g^{A \dot{B}}$ and for the Lorentzian signature $(+++-)$ there must be $\overline{g^{A \dot{B}}}=g^{B \dot{A}}$ where bar stands for the complex conjugation].

More complete treatment of the spinorial formalism in complex relativity see [31,32].

Now we recall some basic facts about the Killing vectors. The system of Killing equations are given by

$$
\nabla_{(a} K_{b)}=\chi g_{a b}
$$


The Killing vector is said to be conformal, if $\chi \neq$ const, homothetic if $\chi=\chi_{0}=$ const $\neq 0$ and isometric if $\chi=0$. A conformal Killing vector $K$ can be written as

$$
K=K^{a} \partial_{a}=-\frac{1}{2} K_{A \dot{B}} \partial^{A \dot{B}}
$$

Components $K^{a}$ and $K_{A \dot{B}}$ are related by

$$
K^{a}=-\frac{1}{2} g^{a A \dot{B}} K_{A \dot{B}} \Longleftrightarrow K_{A \dot{B}}=g_{a A \dot{B}} K^{a}
$$

Conformal Killing equations with conformal factor $\chi(2.7)$ in spinorial form read

$$
\nabla_{A}{ }^{\dot{B}} K_{C}^{\dot{D}}+\nabla_{C}{ }^{\dot{D}} K_{A}^{\dot{B}}=-4 \chi \in_{A C} \in^{\dot{B} \dot{D}}
$$

and this is equivalent to the following system of equations

$$
\begin{aligned}
& \nabla_{(A}^{(\dot{B}} K_{C)}^{\dot{D})}=0 \\
& \nabla^{N \dot{N}} K_{N \dot{N}}+8 \chi=0
\end{aligned}
$$

From (2.11a) and (2.11b) it follows that

$$
\nabla_{A}{ }^{\dot{B}} K_{C} \dot{D}=l_{A C} \in^{\dot{B} \dot{D}}+l^{\dot{B} \dot{D}} \in_{A C}-2 \chi \in_{A C} \in^{\dot{B} \dot{D}}
$$

with

$$
\left.l_{A C}:=\frac{1}{2} \nabla_{(A}^{\dot{N}} K_{C) \dot{N}} \quad l^{\dot{B} \dot{D}}:=\frac{1}{2} \nabla^{N(\dot{B}} K_{N} \dot{D}\right)
$$

The integrability conditions of (2.12) in Einstein space $\left(C_{A B \dot{C} \dot{D}}=0, R=-4 \Lambda\right)$ consist of the following equations

$$
\begin{aligned}
& \nabla_{R}^{\dot{A}} l_{S T}+2 C_{R S T}^{N} K_{N}^{\dot{A}}+\frac{2}{3} \Lambda \in_{R(S} K_{T)}^{\dot{A}}+2 \in_{R(S} \nabla_{T)}^{\dot{A}} \chi=0 \\
& \nabla_{\dot{R}}^{A} l_{\dot{S} \dot{T}}+2 C_{\dot{R} \dot{S} \dot{T}}^{\dot{N}} K_{\dot{N}}^{A}+\frac{2}{3} \Lambda \in_{\dot{R}(\dot{S}} K_{\dot{T})}^{A}+2 \epsilon_{\dot{R}(\dot{S}} \nabla_{\dot{T})}^{A} \chi=0 \\
& K_{N \dot{N}} \nabla^{N \dot{N}} C_{A B C D}+4 C_{(A B C}^{N} l_{D) N}-4 \chi C_{A B C D}=0 \\
& K_{N \dot{N}} \nabla^{N \dot{N}} C_{\dot{A} \dot{B} \dot{C} \dot{D}}+4 C_{(\dot{A} \dot{B} \dot{C}}^{\dot{N}} l_{\dot{D}) \dot{N}}-4 \chi C_{\dot{A} \dot{B} \dot{C} \dot{D}}=0 \\
& \nabla_{A}{ }^{\dot{A}} \nabla_{B}{ }^{\dot{B}} \chi-\frac{2}{3} \Lambda \chi \in_{A B} \in^{\dot{A} \dot{B}}=0 \\
& C^{N}{ }_{A B C} \nabla_{N}{ }^{\dot{A}} \chi=0 \\
& C^{\dot{N}}{ }_{\dot{A} \dot{B} \dot{C}} \nabla_{\dot{N}}^{A} \chi=0
\end{aligned}
$$

[Deep analysis of the Killing equations and their integrability conditions for Killing vectors and Killing tensor fields can be found in [15]]. 


\subsection{Null strings via null Killing vectors}

The existence of a null Killing vector has a significant influence on the geometry of the space. To explain this we first note that the null Killing vector can be presented in the form

$$
K_{A \dot{B}}=\mu_{A} v_{\dot{B}} \Longleftrightarrow K_{A \dot{B}} K^{A \dot{B}}=0
$$

where $\mu_{A}$ and $\nu_{\dot{B}}$ are some nonzero spinors.

Moreover, it is well known that every spinor symmetric in all indices can be decomposed according to the formula

$$
\Psi_{A_{1} A_{2} \ldots A_{n}}=\Psi_{\left(A_{1} A_{2} \ldots A_{n}\right)}=\Psi_{\left(A_{1}\right.}^{(1)} \Psi_{A_{2}}^{(2)} \ldots \Psi_{\left.A_{n}\right)}^{(n)}
$$

where $\Psi_{A}^{(i)}$ are some spinors. In particular, there exist spinors $\mathcal{A}_{A}, \mathcal{B}_{A}, \mathcal{A}_{\dot{A}}$ and $\mathcal{B}_{\dot{A}}$ such that

$$
l_{A B}=\mathcal{A}_{(A} \mathcal{B}_{B)}, \quad l_{\dot{A} \dot{B}}=\mathcal{A}_{(\dot{A}} \mathcal{B}_{\dot{B})}
$$

We prove the following

Lemma 2.1 Spinors $l_{A B}$ and $l_{\dot{A} \dot{B}}$ can be brought to the form $l_{A B}=\mu_{(A} \mathcal{B}_{B)}$ and $l_{\dot{A} \dot{B}}=v_{(\dot{A}} \mathcal{B}_{\dot{B})}$ without any loss of generality.

Proof Inserting (2.15) and (2.17) into (2.12) we obtain

$$
\mu_{C} \nabla_{A}{ }^{\dot{B}} v^{\dot{D}}+v^{\dot{D}} \nabla_{A}{ }^{\dot{B}} \mu_{C}=\mathcal{A}_{(A} \mathcal{B}_{C)} \epsilon^{\dot{B} \dot{D}}+\mathcal{A}^{(\dot{B}} \mathcal{B}^{\dot{D})} \epsilon_{A C}-2 \chi \epsilon_{A C} \epsilon^{\dot{B} \dot{D}}
$$

Contracting (2.18) with $\mu^{A} \mu^{C} v_{\dot{D}}$ one gets

$$
v^{\dot{B}} \mathcal{A}_{A} \mu^{A} \mathcal{B}_{C} \mu^{C}=0
$$

so $\mathcal{A}_{A}$ or $\mathcal{B}_{A}$ must be proportional to $\mu_{A}$. Let $\mathcal{A}_{A}=\mathcal{A} \mu_{A}, \mathcal{A} \neq 0$. Redefining the spinor $\mathcal{B}_{A}$ (absorbing $\mathcal{A}$ into $\mathcal{B}_{A}$ ) we finally get $l_{A B}=\mu_{(A} \mathcal{B}_{B)}$. Analogously we prove that $l_{\dot{A} \dot{B}}=v_{(\dot{A}} \mathcal{B}_{\dot{B})}$.

Before we formulate another theorem, we present some basic facts about the twodimensional holomorphic distributions and their integral manifolds, i.e. null strings.

Let us consider two-dimensional, holomorphic distribution $\mathcal{D}_{\mu^{A}}=\left\{\mu_{A} v_{\dot{B}}, \mu_{A} \rho_{\dot{B}}\right\}$ which is given by the Pfaff system

$$
\mu_{A} g^{A \dot{B}}=0
$$

Distribution $\mathcal{D}_{\mu^{A}}$ is self-dual in the sense, that the 2-form "orthogonal" to the 2-plane belonging to $\mathcal{D}_{\mu^{A}}$ is self-dual. Such a distribution is integrable in the Frobenius sense, if the spinor $\mu_{A}$ satisfies the equations

$$
\mu^{B} \mu^{C} \nabla_{B}^{\dot{A}} \mu_{C}=0
$$


Integral manifolds of the congruence $\mathcal{D}_{\mu^{A}}$ appear to be totally null and geodesic and they are called the null strings. They constitute the congruence of self-dual null strings.

Analogously one can consider anti-self-dual two-dimensional distribution $\mathcal{D}_{v^{\dot{A}}}=$ $\left\{\mu_{A} v_{\dot{B}}, \sigma_{A} v_{\dot{B}}\right\}, \mu_{A} \sigma^{A} \neq 0$ given by the Pfaff system

$$
v_{\dot{B}} g^{A \dot{B}}=0
$$

Such distribution is integrable in the Frobenius sense if $v^{\dot{B}} v^{\dot{C}} \nabla^{A}{ }_{\dot{B}} v_{\dot{C}}=0$ and the integral manifolds are called anti-self-dual null strings.

Null strings are the basic geometrical objects in the theory of heavenly and hyperheavenly spaces. Detailed analysis of the properties of the null strings can be found in $[33,34]$.

Theorem 2.2 Let the null Killing vector $K_{A \dot{B}}$ be of the form (2.15). Then the twodimensional self-dual holomorphic distribution $\left\{\mu_{A} v_{\dot{B}}, \mu_{A} \rho_{\dot{B}}\right\}, v_{\dot{B}} \rho^{\dot{B}} \neq 0$, is integrable and its integral manifolds constitute the congruence of self-dual null strings and the anti-self-dual distribution $\left\{\mu_{A} v_{\dot{B}}, \sigma_{A} v_{\dot{B}}\right\}, \mu_{A} \sigma^{A} \neq 0$, is also integrable and its integral manifolds constitute the congruence of anti-self-dual null strings. Moreover, both Weyl spinors $C_{A B C D}$ and $C_{\dot{A} \dot{B} \dot{C} \dot{D}}$ are algebraically special with $\mu_{A}$ and $\nu_{\dot{B}}$ being the undotted and dotted, respectively, multiple Penrose spinors.

Proof Contracting (2.18) with $\mu^{A} \mu^{C}$ and remembering that $\mathcal{A}_{A}=\mu_{A}$ we get (2.21). This means that the spinor $\mu_{A}$ defines a congruence of self-dual null strings in the sense that the 2-dimensional holomorphic distribution $\left\{\mu_{A} v_{\dot{B}}, \mu_{A} \rho_{\dot{B}}\right\}, v_{\dot{A}} \rho^{\dot{A}} \neq 0$ is integrable and its integrable manifolds constitute the congruence of self-dual null strings. From the complex Sachs-Goldberg theorem it follows [33], that $C_{A B C D}$ is algebraically special and $\mu_{A}$ is multiple Penrose undotted spinor, i.e.

$$
C_{A B C D} \mu^{A} \mu^{B} \mu^{C}=0
$$

Analogously we prove that

$$
v^{\dot{B}} v^{\dot{C}} \nabla_{\dot{B}}^{A} v_{\dot{C}}=0 \quad \text { Sachs-Goldberg theorem } \quad C_{\dot{A} \dot{B} \dot{C} \dot{D}} v^{\dot{A}} v^{\dot{C}} v^{\dot{C}}=0
$$

In particular, from Theorem 2.2 it follows that the integral curves of a null Killing vector are given by the intersection of self-dual and anti-self-dual congruences of null strings.

Note that

$$
\begin{aligned}
\mu^{B} \mu^{C} \nabla_{B}{ }^{\dot{A}} \mu_{C} & =0 \Longleftrightarrow \nabla_{B}{ }^{\dot{A}} \mu_{C}=Z_{B}{ }^{\dot{A}} \mu_{C}+\epsilon_{B C} \Theta^{\dot{A}} \\
v^{\dot{B}} \nu^{\dot{C}} \nabla^{A}{ }_{\dot{B}} \nu_{\dot{C}} & =0 \Longleftrightarrow \nabla_{\dot{B}}^{A} v_{\dot{C}}=X^{A}{ }_{\dot{B}} v_{\dot{C}}+\epsilon_{\dot{B} \dot{C}} \Theta^{A}
\end{aligned}
$$

where $\Theta^{A}$ and $\Theta^{\dot{A}}$ describe the optic properties of the congruence of anti-self-dual and self-dual null strings, respectively. Indeed, if $\Theta^{\dot{A}}=0$ then the self-dual null 
strings are parallely-propagated, if $\Theta^{A}=0$ then anti-self-dual null strings are parallely propagated. Therefore the concept of the expansion of the congruence of the null strings is rather different from the concept of expansion of geodesic congruence in the case of the Lorentzian spacetime. Inserting (2.25), $\mathcal{A}_{A}=\mu_{A}$ and $\mathcal{A}_{\dot{A}}=v_{\dot{A}}$ into (2.18), after some straightforward calculations we obtain

$$
X_{A \dot{B}}=-Z_{A \dot{B}}, \quad \mathcal{B}_{A}=\Theta_{A}, \quad \mathcal{B}_{\dot{A}}=\Theta_{\dot{A}}, \quad \mu_{A} \Theta^{A}+v_{\dot{A}} \Theta^{\dot{A}}+4 \chi=0
$$

We prove another important theorem.

Theorem 2.3 Assume that at least one of the spinors $C_{A B C D}$ or $C_{\dot{A} \dot{B} \dot{C} \dot{D}}$ is nonzero. Then

$$
\begin{gathered}
\text { (i) if } \Lambda \neq 0 \text { then } \quad \chi=0 \\
\text { (ii) if } \Lambda=0 \text { then } \chi=\chi_{0}=\text { const }
\end{gathered}
$$

Proof Assume that $C_{A B C D} \neq 0$. Then from (2.14f) it follows that $\nabla_{A}{ }^{\dot{A}} \chi$ is the quadruple Debever-Penrose spinor. However, as is well known, two quadruple DP spinors are necessarily linearly dependent so $\nabla^{A \dot{1}} \chi$ has to be proportional to $\nabla^{A \dot{2}} \chi$ or, equivalently

$$
\nabla_{A \dot{A}} \chi \cdot \nabla^{A \dot{A}} \chi=0
$$

Acting on (2.27) with $\nabla_{B} \dot{B}$ and using (2.14e) one quickly obtains

$$
\Lambda \chi \nabla_{B}^{\dot{B}} \chi=0
$$

Hence if $\Lambda \neq 0$ then $\nabla_{B} \dot{B} \chi=0$. Finally, using (2.14e) we get $\chi=0$ what proves $(i)$. If $\Lambda=0$, then still $\nabla_{N} \dot{A} \chi$ is a quadruple DP-spinor. However, we proved that $\mu_{N}$ is a multiple DP-spinor, so

$$
\nabla_{N \dot{A}} \chi=\mu_{N} \chi_{\dot{A}}
$$

with some $\chi_{\dot{A}}$. Inserting (2.29) into (2.14e) and contracting with $\mu^{B}$ we arrive at the conclusion $\Theta^{\dot{A}} \chi^{\dot{B}}=0$, so if we want to maintain possible conformal symmetries, the self-dual null string defined by the (conformal) Killing vector must be nonexpanding, $\Theta^{\dot{A}}=0$. Consequently, $l_{\dot{A} \dot{B}}=0$. Inserting this into (2.14b) and contracting with $\in \dot{R} \dot{S}$ we finally get $\chi^{\dot{A}}=0$. From (2.29) it follows that $\nabla_{N \dot{A}} \chi=0$ and this proves (ii).

Summing up, null conformal symmetries can appear only in the Einstein spaces with $C_{A B C D}=0=C_{\dot{A} \dot{B} \dot{C} \dot{D}}$, i.e. in the de-Sitter space (with $\Lambda \neq 0$ ) or in Minkowski space (with $\Lambda=0$ ). We do not consider these spaces here. 
The null Killing vector field defines congruence of null (complex) geodesics. The optical scalars of Killing vector field can be easily obtained. One gets

$$
\begin{aligned}
\frac{1}{2} \nabla^{a} K_{a} & =2 \chi_{0} & \text { (expansion) } \\
\frac{1}{2} \nabla_{[a} K_{b]} \nabla^{[a} K^{b]} & =-2 \chi_{0}^{2} & \text { (twist) } \\
\frac{1}{2} \nabla_{(a} K_{b)} \nabla^{(a} K^{b)}-\frac{1}{4}\left(\nabla^{a} K_{a}\right)^{2} & =-2 \chi_{0}^{2} & \text { (shear) }
\end{aligned}
$$

Thus we conclude that null homothetic Killing field defines null geodesic congruence with nonzero expansion, twist and shear, while null isometric Killing field is nonexpanding, nontwisting and shearfree. It is worth to note, that optical scalars of the null (complex) geodesic congruence defined by $K_{a}$ are related to the expansions of the self-dual congruence of the null strings $\Theta^{\dot{A}}$ and anti-self-dual congruence of the null strings $\Theta^{A}$. This relation is given by the Eqs. (2.30a)-(2.30c) and the crucial condition (2.31f). More general analysis of the optical properties of the congruences of the null strings can be found in [34].

Gathering above considerations: we reduced the problem of null Killing vectors in Einstein space to the set of equations

$$
\begin{aligned}
K_{A \dot{B}}= & \mu_{A} v_{\dot{B}} \\
l_{A B}= & \mu_{(A} \Theta_{B)}, \quad l_{\dot{A} \dot{B}}=v_{(\dot{A}} \Theta_{\dot{B})} \\
\chi_{0}= & \text { const }, \quad \Lambda \chi_{0}=0 \\
\nabla_{A \dot{B}} \mu_{C}= & Z_{A \dot{B}} \mu_{C}+\epsilon_{A C} \Theta_{\dot{B}} \\
\nabla_{A \dot{B}} v_{\dot{C}}= & -Z_{A \dot{B}} v_{\dot{C}}+\epsilon_{\dot{B} \dot{C}} \Theta_{A} \\
& \mu_{A} \Theta^{A}+v_{\dot{A}} \Theta^{\dot{A}}+4 \chi_{0}=0
\end{aligned}
$$

Inserting (2.31b) and (2.31f) into (2.12) we obtain

$$
\nabla_{A}^{\dot{B}} K_{C}^{\dot{D}}=\mu_{C} \Theta_{A} \in^{\dot{B} \dot{D}}+v^{\dot{D}} \Theta^{\dot{B}} \in_{A C}
$$

The formula (2.32) proves, that first covariant derivative of the null Killing vector can be expressed only by the undotted and dotted multiple Penrose spinors and expansions of both self-dual and anti-self-dual congruences of null strings.

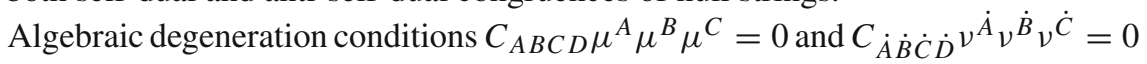
can be combined with (2.14a) and (2.14b). After some work we obtain

$$
\begin{aligned}
& 2 C_{R S T}^{N} \mu_{N}+\Sigma \mu_{R} \mu_{S} \mu_{T}+(3 \Omega+\Lambda) \mu_{(R} \mu_{s} m_{T)}=0 \quad \text { with } m_{A} \mu^{A}=1 \\
& 2 C_{\dot{R} \dot{S} \dot{T}}^{\dot{N}} v_{\dot{N}}+\dot{\Sigma} v_{\dot{R}} v_{\dot{S}} v_{\dot{T}}+(3 \dot{\Omega}+\Lambda) v_{(\dot{R}} v_{\dot{S}} n_{\dot{T})}=0 \quad \text { with } n_{\dot{A}} v^{\dot{A}}=1
\end{aligned}
$$


where $\Sigma, \dot{\Sigma}, \Omega$ and $\dot{\Omega}$ are defined by the relations

$$
\begin{aligned}
& \nabla_{R}^{\dot{A}}\left(\mu_{(S} \Theta_{T)}\right)=v^{\dot{A}}\left(\Sigma \mu_{R} \mu_{S} \mu_{T}+2 \Omega \mu_{R} \mu_{\left(S m_{T)}\right.}+(\Lambda+\Omega) \mu_{S} \mu_{T} m_{R}\right) \\
& \nabla_{R}^{\dot{A}}\left(v_{(\dot{S}} \Theta_{\dot{T})}\right)=\mu_{R}\left(\dot{\Sigma} v^{\dot{A}} v_{\dot{S}} v_{\dot{T}}+2 \dot{\Omega} v^{\dot{A}} v_{(\dot{S}} n_{\dot{T})}+(\Lambda+\dot{\Omega}) n^{\dot{A}} v_{\dot{S}} v_{\dot{T}}\right)
\end{aligned}
$$

We end this subsection by pointing out two relations essential in further analysis. Contracting (2.34a) with $\mu_{S} \mu_{T}$ and using (2.31d) we obtain

$$
\Theta^{\dot{A}} \mu_{S} \Theta^{S}=0
$$

Analogously, contracting (2.34b) with $v_{\dot{S}} v_{\dot{T}}$ and using (2.31e) one gets

$$
\Theta^{A} v_{\dot{S}} \Theta^{\dot{S}}=0
$$

Now we are ready to discuss the possible algebraic types admitting null Killing vector. Note, that we follow Petrov-Penrose algebraic classification of the Weyl spinors $C_{A B C D}$ and $C_{\dot{A} \dot{B} \dot{C} \dot{D}}$ in complex spacetimes $[32,8,13,14]$. In real relativity with Lorentzian signature $(+++-)$ the algebraic type of the undotted Weyl spinor is the same, as the type of dotted Weyl spinor. However, in complex specetimes spinors $C_{A B C D}$ and $C_{\dot{A} \dot{B} \dot{C} \dot{D}}$ are unrelated. Hence, the "mixed" types (like [N] $\otimes[\mathrm{D}]$ or $[\mathrm{II}] \otimes[\mathrm{III}])$ can appear.

\subsection{Null homothetic symmetries}

Here we assume $\chi_{0} \neq 0$, what immediately gives $\Lambda=0$. Simple analysis of Eqs. (2.35)-(2.36) together with (2.31f) brings us to the conclusion that the only possibilities are

- $\Theta^{\dot{A}}=0$ (the congruence of self-dual null strings is nonexpanding), $\mu_{A} \Theta^{A} \neq 0$ (the congruence of anti-self-dual null strings is necessarily expanding, more even, expansion $\Theta^{A}$ cannot be proportional to DP-spinor $\mu^{A}$ )

- $\Theta^{A}=0$ (the congruence of anti-self-dual null strings is nonexpanding), $v_{\dot{A}} \Theta^{\dot{A}} \neq 0$ (the congruence of self-dual null strings is necessarily expanding, more even, expansion $\Theta^{\dot{A}}$ cannot be proportional to DP-spinor $\nu^{\dot{A}}$ )

Of course, both possibilities constitute Eintein spaces with the same geometric properties. It is enough to consider only one of them with details, say $\Theta^{\dot{A}}=0$. From (2.34b) we conclude that $\dot{\Sigma}=\dot{\Omega}=0$. Careful analysis of (2.34a) gives $\Omega=0$. From (2.33a) and (2.33b) we obtain

$$
\begin{aligned}
& 2 C_{R S T}^{N} \mu_{N}+\Sigma \mu_{R} \mu_{S} \mu_{T}=0 \\
& 2 C_{\dot{R} \dot{S} \dot{T}}^{\dot{N}} \nu_{\dot{N}}=0
\end{aligned}
$$


where

$$
\Sigma \mu_{R} \mu_{T} v_{\dot{A}}:=\nabla_{R \dot{A}} \Theta_{T}+\Theta_{T} Z_{R \dot{A}}
$$

(the last formula is a consequence of (2.34a).

The only possible anti-self-dual Petrov types are $[\mathrm{N},-]$. From $(2.37 \mathrm{a})$ we easily get that the only possible self-dual Petrov types are [III, - ]; self-dual type [N] is not admitted. Indeed assume, that $C_{A B C D}$ is of the type [N], so $C_{R S T}^{N} \mu_{N}=0$ what gives $\Sigma=0$. Contracting (2.38) with $\Theta^{T}$ we obtain $\Theta^{T} \nabla_{R \dot{A}} \Theta_{T}=0$, so $\Theta^{T}$ defines the congruence of self-dual null strings. But we proved earlier (see Theorem 2.2), that the self-dual null string is defined by $\mu^{T}$. The number of independent congruences of self-dual null strings is equal to the number of multiple undotted DP-spinors, so there are infinitely many independent congruences of self-dual null strings in the heavenly spaces, two for the self-dual type [D] and only one for the self-dual types [II, III, N]. But here we examine self-dual type $[\mathrm{N}]$, so there is only one congruence of the nullstrings. It means, that $\Theta^{T}$ must be proportional to $\mu^{T}$ or $\Theta^{T} \mu_{T}=0 \Longrightarrow \chi_{0}=0$. This contradicts our assumption that $\chi_{0} \neq 0$ and proves that the only possible Petrov types which admit null homothetic symmetries are $[\mathrm{III},-] \otimes[\mathrm{N},-]$. Then the congruence of self-dual null strings is nonexpanding and the congruence of anti-self-dual null strings must be expanding.

Remark considering the second possibility with $\Theta^{A}=0$ we obtain possible Petrov types $[\mathrm{N},-] \otimes[\mathrm{III},-]$, but still type [III] admits the congruence of nonexpanding null strings and the type $[\mathrm{N}]$ admits the expanding congruence.

All possible types via geometric properties of the congruence of null strings are presented in the table below:

\begin{tabular}{lll}
\hline & $\Theta^{\dot{A}}=0$ & $\Theta^{\dot{A}} \neq 0$ \\
\hline$\Theta^{A}=0$ & Not admitted & {$[\mathrm{N},-]^{e} \otimes[\mathrm{III},-]^{n}$} \\
$\Theta^{A} \neq 0$ & {$[\mathrm{III},-]^{n} \otimes[\mathrm{N},-]^{e}$} & not admitted \\
\hline
\end{tabular}

The upper index $e$ means, that the corresponding congruence of null strings is expanding, index $n$ - nonexpanding.

\subsection{Null isometric symmetries}

Here we assume $\chi_{0}=0$. Analysis of Eqs. (2.35), (2.36) and (2.31f) leads to the conclusion that $\Theta^{A}$ and $\Theta^{\dot{A}}$ must have the form

$$
\Theta^{A}=\Theta \mu^{A}, \quad \Theta^{\dot{A}}=\dot{\Theta} v^{\dot{A}}
$$

(dot in $\dot{\Theta}$ does not mean derivative here). Hence

$$
l_{A B}=\Theta \mu_{A} \mu_{B}, \quad l_{\dot{A} \dot{B}}=\dot{\Theta} v_{\dot{A}} v_{\dot{B}}
$$


Using (2.39) and (2.40) in (2.34a) and (2.34b) we find $\Omega=\dot{\Omega}=\Theta \dot{\Theta}$. Equations (2.33a) and (2.33b) can be rearranged to the form

$$
\begin{aligned}
& 2 C_{R S T}^{N} \mu_{N}+\Sigma \mu_{S} \mu_{R} \mu_{T}+(\Lambda+3 \Theta \dot{\Theta}) \mu_{(S} \mu_{R} m_{T)}=0 \\
& 2 C_{\dot{R} \dot{S} \dot{T}}^{\dot{N}} v_{\dot{N}}+\dot{\Sigma} v_{\dot{S}} v_{\dot{R}} v_{\dot{T}}+(\Lambda+3 \Theta \dot{\Theta}) v_{(\dot{S}} v_{\dot{R}} n_{\dot{T})}=0
\end{aligned}
$$

and the Eqs. (2.34a) and (2.34b) read

$$
\begin{aligned}
& \nabla_{R \dot{A}} \Theta+2 \Theta Z_{R \dot{A}}=: v_{\dot{A}}\left(\Sigma \mu_{R}+(\Lambda+3 \Theta \dot{\Theta}) m_{R}\right) \\
& \nabla_{R \dot{A}} \dot{\Theta}-2 \dot{\Theta} Z_{R \dot{A}}=: \mu_{R}\left(\dot{\Sigma} v_{\dot{A}}+(\Lambda+3 \Theta \dot{\Theta}) n_{\dot{A}}\right)
\end{aligned}
$$

Multiplying (2.42a) by $\dot{\Theta}$ and (2.42b) by $\Theta$ and adding both equations one arrives at the useful formula

$$
\frac{1}{3} \nabla_{R \dot{A}}(\Lambda+3 \Theta \dot{\Theta})=(\Sigma \dot{\Theta}+\dot{\Sigma} \Theta) \mu_{R} v_{\dot{A}}+(\Lambda+3 \Theta \dot{\Theta})\left(\Theta \mu_{R} n_{\dot{A}}+\dot{\Theta} m_{R} v_{\dot{A}}\right)
$$

When both congruences of null strings are nonexpanding $(\Theta=\dot{\Theta}=0)$ then from (2.42a) and (2.42b) it follows that $\Lambda=\Sigma=\dot{\Sigma}=0$. Consequently, from (2.41a) and (2.41b) we infer that the only possible types are $[\mathrm{N},-] \otimes[\mathrm{N},-]$.

If the congruence of anti-self-dual null strings is nonexpanding $(\Theta=0)$ and the congruence of self-dual null strings is expanding $(\dot{\Theta} \neq 0)$ then from $(2.42 \mathrm{a})$ we get $\Lambda=\Sigma=0$, so the self-dual type is at most of the type [N]. The anti-self-dual type can be of the type [III] (if $\dot{\Sigma} \neq 0$ ) or of the type [N, - ] (if $\dot{\Sigma}=0$ ), so in this case we deal with the types $[\mathrm{N},-] \otimes[\mathrm{III}, \mathrm{N},-]$. [The case with expanding congruence of anti-self-dual strings and nonexpanding congruence of self-dual strings has the same geometry and leads to the types [III,N, - ] $\otimes[\mathrm{N},-]$.

In the last case both congruences of null strings are expanding $\Theta \neq 0, \dot{\Theta} \neq 0$. Equations (2.41a) and (2.41b) give in general types [II,D] $\otimes$ [II,D]. Cosmological constant $\Lambda$ can be arbitrary here. [It does not follow from (2.41a) - (2.41b), but the mixed types $[\mathrm{II}] \otimes[\mathrm{D}]$ and $[\mathrm{D}] \otimes[\mathrm{II}]$ are not admitted, we will prove this during further analysis].

Now we deal with the self-dual type [III], $C_{A B C D} \mu^{C} \mu^{D}=0$. From (2.41a) we got $\Lambda+3 \Theta \dot{\Theta}=0$ (so the cosmological constant $\Lambda$ is necessarily nonzero), from (2.41b) we conclude, that the anti-self-dual type is [III]. Indeed, anti-self-dual types [N, - ] imply $\dot{\Sigma}=0$, what combined with (2.43) gives $\Sigma=0$. But $\Sigma=0$ automatically reduces the self-dual type to $[\mathrm{N},-]$.

In self-dual types [N, - ], $C_{A B C D} \mu^{D}=0$ so $\Sigma=0=\Lambda+3 \Theta \dot{\Theta}$. Immediately we have $\dot{\Sigma}=0$, so anti-self-dual type is $[\mathrm{N},-]$ too. Like in previous case, cosmological constant $\Lambda$ must be nonzero here.

All possible types are gathered in the table below: 


\begin{tabular}{lll}
\hline & $\dot{\Theta}=0$ & $\dot{\Theta} \neq 0$ \\
\hline$\Theta=0$ & {$[\mathrm{~N},-]^{n} \otimes[\mathrm{N},-]^{n}, \Lambda=0$} & {$[\mathrm{~N},-]^{e} \otimes[\mathrm{III}, \mathrm{N},-]^{n}, \Lambda=0$} \\
$\Theta \neq 0$ & {$[\mathrm{III}, \mathrm{N},-]^{n} \otimes[\mathrm{N},-]^{e}, \Lambda=0$} & {$[\mathrm{II}]^{e} \otimes[\mathrm{II}]^{e},[\mathrm{D}]^{e} \otimes[\mathrm{D}]^{e}, \Lambda$ arbitrary } \\
& & {$[\mathrm{III}]^{e} \otimes[\mathrm{III}]^{e}, \Lambda \neq 0$} \\
& {$[\mathrm{~N},-]^{e} \otimes[\mathrm{N},-]^{e}, \Lambda \neq 0$} \\
\hline
\end{tabular}

\section{Hyperheavenly spaces}

The considerations from the previous section allow us to establish all possible algebraic types of the spaces which admit the null Killing symmetry. Main aim of the present paper is to find the explicit metrics with such symmetries. Due to Theorem 2.2, any null Killing vector defines the congruences of both self-dual and anti-self-dual null strings and implies the algebraic degeneration of both self-dual and anti-self-dual part of the Weyl curvature spinor. Let us remind the definition of hyperheavenly space $[10,12-14]$.

Definition 3.1 Hyperheavenly space $(\mathcal{H} \mathcal{H}$-space) with cosmological constant is a 4-dimensional complex analytic differential manifold endowed with a holomorphic Riemannian metric $d s^{2}$ satisfying the vacuum Einstein equations with cosmological constant and such that the self-dual or anti-self-dual part of the Weyl tensor is algebraically degenerate. These kind of spaces admits a congruence of totally null, self-dual (or anti-self-dual, respectively) surfaces.

A complex 4-dimensional space which admits the null Killing vector is equipped with both self-dual and anti-self-dual congruences of null strings. If, moreover, such a space is Einstein then by the Goldberg-Sachs theorem it is algebraically special for both sides and of course is a $\mathcal{H} \mathcal{H}$-space. Vacuum Einstein equations in $\mathcal{H} \mathcal{H}$-space can be reduced to one, nonlinear, partial differential equation of the second order, for one holomorphic function. This equation is called hyperheavenly equation.

The existence of the null strings allows us to introduce some useful tetrad and the coordinate system. The self-dual null string generated by the null Killing vector is given by the equation $\mu^{B} \mu^{C} \nabla_{B} \dot{A} \mu_{C}=0$ which is equivalent to the Pfaff system $\mu_{A} g^{A \dot{B}}=0$. Choosing the spinorial basis in such a manner that $\mu_{A}=\left(0, \mu_{2}\right), \mu_{2} \neq 0$ we arrive at the conclusion, that the congruence of self-dual null strings is defined by the Pfaff system

$$
e^{1}=0, \quad e^{3}=0 \Longleftrightarrow g^{2 \dot{A}}=0
$$

(the surface element of the null string is given by $e^{1} \wedge e^{3}$ ).

A null tetrad $\left(e^{1}, e^{2}, e^{3}, e^{4}\right)$ and a coordinate system $\left(q_{\dot{A}}, p^{\dot{B}}\right)$ can be always chosen so that $[13,10,14]$

$$
\begin{aligned}
-\frac{1}{\sqrt{2}} g^{2}{ }_{\dot{A}} & =\left[\begin{array}{l}
e^{3} \\
e^{1}
\end{array}\right]=\phi^{-2} d q_{\dot{A}} \\
\frac{1}{\sqrt{2}} g^{1 \dot{A}} & =\left[\begin{array}{l}
e^{4} \\
e^{2}
\end{array}\right]=-d p^{\dot{A}}+Q^{\dot{A} \dot{B}} d q_{\dot{B}}
\end{aligned}
$$


where $\phi$ and $Q^{\dot{A} \dot{B}}=Q^{\dot{B} \dot{A}}$ are holomorphic functions. Coordinates $q_{\dot{A}}$ label the null strings and $p^{\dot{A}}$ are coordinates on them. Dual basis is given by

$$
-\partial_{\dot{A}}=\left[\begin{array}{c}
\partial_{4} \\
\partial_{2}
\end{array}\right], \quad \partial^{\dot{A}}=\left[\begin{array}{l}
\partial_{3} \\
\partial_{1}
\end{array}\right], \quad \partial^{A \dot{B}}=\sqrt{2}\left(\delta_{1}^{A} \partial^{\dot{B}}-\delta_{2}^{A} \partial^{\dot{B}}\right)
$$

where

$$
\partial_{\dot{A}}:=\frac{\partial}{\partial p^{\dot{A}}}, \quad \partial^{\dot{A}}:=\phi^{2}\left(\frac{\partial}{\partial q_{\dot{A}}}+Q^{\dot{A} \dot{B}} \partial_{\dot{B}}\right)
$$

Of course, for consistency with (2.3), the rules to raise and lower spinor indices in spinorial differential operators read $\partial^{\dot{A}}=\partial_{\dot{B}} \epsilon^{\dot{A} \dot{B}}, \partial_{\dot{A}}=\epsilon_{\dot{B} \dot{A}} \partial^{\dot{B}}, \check{\partial}^{\dot{A}}=\partial_{\dot{B}} \epsilon^{\dot{A} \dot{B}}$, $\partial_{\dot{A}}=\epsilon_{\dot{B} \dot{A}} \partial^{\dot{B}}$, so

$$
\partial^{\dot{A}}=\frac{\partial}{\partial p_{\dot{A}}}, \quad \partial_{\dot{A}}=\phi^{2}\left(\frac{\partial}{\partial q^{\dot{A}}}-Q_{\dot{A}}^{\dot{B}} \partial_{\dot{B}}\right)
$$

The metric $d s^{2}$ is given by

$d s^{2}=2 \underset{S}{1} \underset{s}{\otimes} e^{2}+2 e^{3} \underset{s}{\otimes} e^{4}=-\frac{1}{2} g_{A \dot{B}} \underset{S}{\otimes} g^{A \dot{B}}=2 \phi^{-2}\left(-d p^{\dot{A}} \underset{S}{\otimes} d q_{\dot{A}}+Q^{\dot{A} \dot{B}} d q_{\dot{A}} \otimes_{S} d q_{\dot{B}}\right)$

The congruence of null strings have some invariant properties. Investigating the equation $\nabla_{B} \dot{A} \mu_{C}=Z_{B} \dot{A} \mu_{C}+\epsilon_{B C} \Theta^{\dot{A}}$ with $\mu_{1}=0, \mu_{2} \neq 0$ we easily find, that

$$
\begin{aligned}
\Theta_{\dot{A}} & =\Gamma_{112 \dot{A}} \mu_{2} \\
Z_{A \dot{B}} & =-\Gamma_{12 A \dot{B}}-\epsilon_{A 2} \Gamma_{112 \dot{B}}+\partial_{A \dot{B}} \ln \mu_{2}
\end{aligned}
$$

If $\Theta^{\dot{A}}=0 \Longleftrightarrow \Gamma_{112 \dot{A}}=0$ then self-dual null strings are parallely propagated. The hyperheavenly spaces based on such congruence of null strings are called nonexpanding. If the null strings are not parallely propagated $\left(\Theta^{\dot{A}} \neq 0 \Longleftrightarrow \Gamma_{112 \dot{A}} \neq 0\right)$, the corresponding hyperheavenly space is called expanding.

Vacuum Einstein equations impose some constraints on $\phi$ and $Q^{\dot{A} \dot{B}}$. The final forms of the $\phi$ and $Q^{\dot{A} \dot{B}}$ are esentially different for expanding and nonexpanding hyperheavenly spaces.

Nonexpanding hyperheavenly spaces

If $\Theta^{\dot{A}}=0$ the $\phi$ and $Q^{\dot{A} \dot{B}}$ can be brought to the following forms

$$
\phi=1, \quad Q^{\dot{A} \dot{B}}=-\theta_{p_{\dot{A}} p_{\dot{B}}}+\frac{2}{3} F^{(\dot{A}} p^{\dot{B})}+\frac{1}{3} \Lambda p^{\dot{A}} p^{\dot{B}}
$$


where $\theta_{p^{\dot{B}}}:=\frac{\partial \theta}{\partial p^{\dot{B}}}$, etc.. The Einstein equations can be reduced to the nonexpanding hyperheavenly equation with $\Lambda$

$$
\begin{aligned}
& \frac{1}{2} \theta_{p_{\dot{A}} p_{\dot{B}}} \theta_{p^{\dot{A}} p^{\dot{B}}}+\theta_{p_{\dot{A}} q^{\dot{A}}}+F^{\dot{A}}\left(\theta_{p^{\dot{A}}}-\frac{2}{3} p^{\dot{B}} \theta_{p^{\dot{A}} p^{\dot{B}}}\right)+\frac{1}{18}\left(F^{\dot{A}} p_{\dot{A}}\right)^{2} \\
& +\frac{1}{6} \frac{\partial F_{\dot{A}}}{\partial q^{\dot{B}}} p^{\dot{A}} p^{\dot{B}}+\Lambda\left(p^{\dot{A}} \theta_{p^{\dot{A}}}-\theta-\frac{1}{3} p^{\dot{A}} p^{\dot{B}} \theta_{p^{\dot{A}} p^{\dot{B}}}\right)=N_{\dot{A}} p^{\dot{A}}+\gamma
\end{aligned}
$$

where $F^{\dot{A}}, N^{\dot{A}}$ and $\gamma$ are arbitrary functions of $q^{\dot{C}}$ only (constant on each self-dual null string), $\Lambda$ is a cosmological constant and $\theta=\theta\left(p^{\dot{A}}, q_{\dot{B}}\right)$ is the key function. The metric is defined by (3.6) with $\phi$ and $Q^{\dot{A} \dot{B}}$ given by (3.9).

\section{Expanding hyperheavenly spaces}

If the congruence of self-dual null strings is expanding, we obtain

$$
\phi=J_{\dot{A}} p^{\dot{A}}, \quad Q^{\dot{A} \dot{B}}=-2 J^{(\dot{A}} W_{p_{\dot{B})}}-\phi W_{p_{\dot{A}} p_{\dot{B}}}+\frac{1}{\tau^{2}} K^{\dot{A}} K^{\dot{B}}\left(\mu \phi^{3}+\frac{\Lambda}{6}\right)
$$

where $\mu=\mu\left(q^{\dot{N}}\right)$ is an arbitrary function, $\Lambda$ is the cosmological constant, $W=$ $W\left(p^{\dot{A}}, q_{\dot{B}}\right)$ is the key function and $J_{\dot{A}}$ and $K_{\dot{A}}$ are constant, nonzero spinors, satisfying the relation

$$
K^{\dot{A}} J_{\dot{B}}-K_{\dot{B}} J^{\dot{A}}=\tau \delta_{\dot{B}}^{\dot{A}} \quad \text { where } \quad \tau=K^{\dot{A}} J_{\dot{A}} \neq 0
$$

[ $\tau$ is an arbitrary constant; not loosing generality one can set $\tau=1$.]

Einstein equations can be reduced to the expanding hyperheavenly equation with $\Lambda$

$$
\begin{gathered}
\frac{1}{2} \phi^{4}\left(\phi^{-2} W_{p_{\dot{B}}}\right)_{p_{\dot{A}}}\left(\phi^{-2} W_{p^{\dot{B}}}\right)_{p^{\dot{A}}}+\phi^{-1} W_{p_{\dot{A}} q^{\dot{A}}}-\mu \phi^{4}\left[\phi^{-1}\left(\phi^{-1} W\right)_{\phi}\right]_{\phi} \\
+\frac{\eta}{2 \tau^{2}}\left(\eta J^{\dot{C}}-\phi K^{\dot{C}}\right) \mu_{q^{\dot{C}}}-\frac{\Lambda}{6} \phi^{-1} W_{\phi \phi}=N_{\dot{A}} p^{\dot{A}}+\gamma
\end{gathered}
$$

where $N^{\dot{A}}$ and $\gamma$ are arbitrary functions of $q^{\dot{C}}$ only (constant on each self-dual null string). Instead of the $\left(p^{\dot{A}}, q_{\dot{B}}\right)$-coordinate system, another one, namely $(\phi, \eta, w, t)$ is universally used

$$
\begin{aligned}
& \phi=J_{\dot{A}} p^{\dot{A}}, \quad \eta:=K^{\dot{A}} p_{\dot{A}} \Longleftrightarrow \tau p^{\dot{A}}=\eta J^{\dot{A}}+\phi K^{\dot{A}} \\
& w:=J_{\dot{A}} q^{\dot{A}}, \quad t:=K^{\dot{A}} q_{\dot{A}} \Longleftrightarrow \tau q_{\dot{A}}=t J_{\dot{A}}+w K_{\dot{A}}
\end{aligned}
$$

with the operators

$$
\partial_{\phi}=\frac{1}{\tau} K^{\dot{A}} \partial_{\dot{A}}, \quad \partial_{\eta}=\frac{1}{\tau} J^{\dot{A}} \partial_{\dot{A}}, \quad \partial_{w}=\frac{1}{\tau} K^{\dot{A}} \frac{\partial}{\partial q^{\dot{A}}}, \quad \partial_{t}=\frac{1}{\tau} J^{\dot{A}} \frac{\partial}{\partial q^{\dot{A}}}
$$


In $(\phi, \eta, w, t)$-language, the hyperheavenly equation reads

$$
\begin{gathered}
\tau^{2}\left(W_{\eta \eta} W_{\phi \phi}-W_{\eta \phi} W_{\eta \phi}+2 \phi^{-1} W_{\eta} W_{\eta \phi}-2 \phi^{-1} W_{\phi} W_{\eta \eta}\right)+\tau \phi^{-1}\left(W_{w \eta}-W_{t \phi}\right) \\
-\mu\left(\phi^{2} W_{\phi \phi}-3 \phi W_{\phi}+3 W\right)+\frac{\eta}{2 \tau}\left(\mu_{t} \eta-\mu_{w} \phi\right)-\frac{\Lambda}{6} \phi^{-1} W_{\phi \phi}=\frac{1}{2} \varkappa \phi-\frac{1}{2} \nu \eta+\gamma
\end{gathered}
$$

where $2 N_{\dot{A}}=: v K_{\dot{A}}+\varkappa J_{\dot{A}}$.

The metric (3.6) takes now the form

$$
\begin{aligned}
d s^{2}= & (\phi \tau)^{-2}\left\{2 \tau(d \eta \underset{s}{\otimes} d w-d \phi \underset{s}{\otimes} d t)+2\left(-\tau^{2} \phi W_{\eta \eta}+\mu \phi^{3}+\frac{\Lambda}{6}\right) d t \underset{s}{\otimes} d t\right. \\
& \left.+4\left(-\tau^{2} \phi W_{\eta \phi}+\tau^{2} W_{\eta}\right) d w \underset{s}{\otimes} d t+2\left(-\tau^{2} \phi W_{\phi \phi}+2 \tau^{2} W_{\phi}\right) d w \underset{s}{\otimes} d w\right\}
\end{aligned}
$$

We do not present here the curvature formulas and connection forms. The reader is referred to $[12,25,26]$. We note only that the expansion of the congruence of the selfdual null strings is proportional to nonzero spinor $J_{\dot{A}}$, namely $\Theta_{\dot{A}}=-\sqrt{2} \phi^{-1} \mu_{2} J_{\dot{A}}$.

\section{Metrics admitting null homothetic symmetries}

\subsection{Hyperheavenly spaces of the type $[\mathrm{N}]^{e} \otimes[\mathrm{III}]^{n}$}

The hyperheavenly spaces which admit the null homothetic symmetry must be of types $[\mathrm{N}]^{e} \otimes[\mathrm{III}]^{n}$ or $[\mathrm{III}]^{n} \otimes[\mathrm{N}]^{e}$. Such spaces have been considered previously in [29] and [28] but without any details. Since the type $[\mathrm{III}]^{n} \otimes[\mathrm{N}]^{e}$ can be obtained from $[\mathrm{N}]^{e} \otimes[\mathrm{III}]^{n}$ just by changing the orientation we study the case $[\mathrm{N}]^{e} \otimes[\mathrm{III}]^{n}$ and use the general formulas from [29]. Killing vector has the form

$$
K=-2 \chi_{0} p^{\dot{A}} \frac{\partial}{\partial p^{\dot{A}}}
$$

and

$$
l_{A B}=0, \quad l^{\dot{A} \dot{B}}=4 \chi_{0} \phi^{-1} J^{(\dot{A}} p^{\dot{B})}
$$

The key function and the curvature read

$$
\begin{aligned}
& W=\phi^{2} F(x, w, t), \quad x:=\frac{\eta}{\phi} \\
& C^{(1)}=2 \phi^{7} \tau \gamma_{t}, \quad C_{\dot{A} \dot{B} \dot{C} \dot{D}}=C_{(\dot{A}} p_{\dot{B}} p_{\dot{C}} p_{\dot{D})} \\
& C_{\dot{A}}:=4 \tau^{3} \phi^{-2} F_{x x x} J_{\dot{A}}+\tau^{4} \phi^{-3} F_{x x x x} p_{\dot{A}}
\end{aligned}
$$


where $F=F(x, w, t)$ and $\gamma=\gamma(w, t)$ are arbitrary functions of their arguments such that $F_{x x x} \neq 0$ and $\gamma_{t} \neq 0$. Inserting the key function $W$ (4.3) into hyperheavenly equation we get

$$
F_{x}^{2}-2 F F_{x x}+F_{x w^{\prime}}+x F_{x t^{\prime}}-2 F_{t^{\prime}}=\gamma^{\prime}
$$

where $w^{\prime}=\tau w, t^{\prime}=\tau t$ and $\gamma^{\prime}=\tau^{-2} \gamma$. The general solution of the Eq. (4.5) is not known. The metric has the form

$$
\begin{aligned}
d s^{2}= & 2 \phi^{-2}\left\{\tau^{-1}(d \eta \underset{s}{\otimes} d w-d \phi \underset{s}{\otimes} d t)-\phi F_{x x} d t \underset{s}{\otimes} d t\right. \\
& \left.+2 \phi x F_{x x} d w \underset{s}{\otimes} d t+\phi\left(2 F-x^{2} F_{x x}\right) d w \underset{s}{\otimes} d w\right\}, \quad \eta=x \phi
\end{aligned}
$$

where $F=F(x, w, t)$ satisfies the Eq. (4.5).

\subsection{Heavenly spaces of the type $[\mathrm{N}]^{e} \otimes[-]^{n}$}

There are two, essentially different heavenly reductions of the hyperheavenly space of the type $[\mathrm{N}]^{e} \otimes[\mathrm{III}]^{n}$ with null homothetic symmetry. Taking $F_{x x x}=0 \Longleftrightarrow$ $C_{\dot{A}}=0 \Longleftrightarrow C_{\dot{A} \dot{B} \dot{C} \dot{D}}=0$ in (4.4) we obtain the space of the type $[\mathrm{N}]^{e} \otimes[-]^{n}$ with expanding self-dual null strings.

Equation (4.5) under the additional assumption $F_{x x x}=0$ can be easily solved. Using gauge freedom which is still available (see [29] for details) one gets

$$
\begin{aligned}
& W=\frac{1}{2} f(w, t) \phi^{2} \\
& C^{(1)}=-2 \phi^{7} f_{t t}
\end{aligned}
$$

where $f=f(w, t)$ is an arbitrary function. The metric is

$$
d s^{2}=\frac{2}{\tau} \phi^{-2}(d \eta \underset{s}{\otimes} d w-d \phi \underset{s}{\otimes} d t+\tau f \phi d w \underset{s}{\otimes} d w)
$$

\subsection{Heavenly spaces of the type $[\mathrm{III}]^{n} \otimes[-]^{e}$}

The second possible heavenly reduction of the hyperheavenly space of the type $[\mathrm{N}]^{e} \otimes$ $[\mathrm{III}]^{n}$ with null homothetic symmetry is the heavenly space of the type $[-]^{e} \otimes[\mathrm{III}]^{n}$. Formally it is enough to set $C^{(1)}=0 \Longleftrightarrow \gamma_{t}=0$ in subsection 4.1 . However, the Eq. (4.5) is still hard to solve. It appears to be much more convenient to attack the problem from the opposite side and consider the space of the type $[\mathrm{III}]^{n} \otimes[-]^{e}$. As a starting point we take the nonexpanding hyperheavenly spaces and we set $C_{\dot{A} \dot{B} \dot{C} \dot{D}}=0$. It allows us to take the general key function as a third-order polynomial in $p^{\dot{A}}$ coordinates (see $[27,28]$ for details). The metric appears to be two-sided Walker [25]. Using the general results from [28] and [25] (especially Theorem 5.1 from [25]) we find the form 
of the Killing vector

$$
K=2 \chi_{0} p^{\dot{A}} \frac{\partial}{\partial p^{\dot{A}}}
$$

and the spinors $l_{A B}$ and $l_{\dot{A} \dot{B}}$

$$
l_{A B}=-4 \chi_{0} \delta_{(A}^{1} \delta_{B)}^{2}, \quad l_{\dot{A} \dot{B}}=0
$$

The key function and the curvature read

$$
\begin{aligned}
& \theta=\frac{1}{6} p^{\mathrm{i}} p^{\dot{M}}\left(p^{2} \frac{\partial X}{\partial q^{\dot{M}}}-p^{\mathrm{i}} e^{X} \frac{\partial Y}{\partial q^{\dot{M}}}\right) \\
& C^{(2)}=2 \frac{\partial}{\partial q_{\dot{j}}}\left(e^{X} \frac{\partial Y}{\partial q_{\dot{1}}}-\frac{\partial X}{\partial q_{\dot{2}}}\right), \quad C^{(1)}=p^{\dot{A}}\left(F_{\dot{A}} C^{(2)}+\frac{\partial C^{(2)}}{\partial q^{\dot{A}}}\right) \\
& \text { where } F_{\dot{1}}=\frac{\partial X}{\partial q_{\dot{2}}}-2 e^{X} \frac{\partial Y}{\partial q_{\dot{j}}}, \quad F_{\dot{2}}=\frac{\partial X}{\partial q_{\dot{1}}}
\end{aligned}
$$

where $X=X\left(q^{\dot{M}}\right)$ and $Y=Y\left(q^{\dot{M}}\right)$ are arbitrary functions. The metric is

$$
\begin{aligned}
& d s^{2}=2\left(-d p^{\dot{A}} \otimes_{s} d q_{\dot{A}}+Q^{\dot{A} \dot{B}} d q_{\dot{A}} \underset{s}{\otimes} d q_{\dot{B}}\right) \\
& Q^{\dot{1} \dot{1}}=-p^{\dot{1}} \frac{\partial X}{\partial q^{\dot{2}}}, \quad Q^{\dot{1} \dot{2}}=-p^{\dot{1}} e^{X} \frac{\partial Y}{\partial q^{\dot{2}}}, \\
& Q^{\dot{2} \dot{2}}=-p^{\dot{2}} \frac{\partial X}{\partial q^{\dot{1}}}-p^{\dot{2}} e^{X} \frac{\partial Y}{\partial q^{\dot{2}}}+p^{\dot{1}} e^{X} \frac{\partial Y}{\partial q^{\dot{1}}}
\end{aligned}
$$

[Note, that the conditions $C_{\dot{A} \dot{B} \dot{C} \dot{D}}=0=\Lambda$ assure the existence of infinitely many congruences of nonexpanding anti-self-dual null strings, that is why the space considered belongs to the two-sided Walker class. However, the congruence of anti-self-dual null strings generated by the null Killing vector is expanding.]

\section{Metrics admitting null isometric symmetries}

5.1 Spaces of the type $[\mathrm{II}]^{e} \otimes[\mathrm{III}]^{e}$

\subsubsection{General case $\Lambda \neq 0$}

If both congruences of null strings are expanding then null isometric symmetries are admitted by the types $[\mathrm{II}] \otimes[\mathrm{II}]$ and $[\mathrm{D}] \otimes[\mathrm{D}]$. The respective metrics have been discussed in [18] (with $\Lambda=0$ ) and then in [29] (with $\Lambda \neq 0$ ). The Killing vector reads

$$
K=\frac{\partial}{\partial \eta}
$$


and

$$
l_{A B}=\frac{2 \phi}{\tau}\left(\mu_{0} \phi^{3}+\frac{\Lambda}{6}\right) \delta_{(A}^{2} \delta_{B)}^{2}, \quad l^{\dot{A} \dot{B}}=-\frac{2}{\tau} \phi^{-1} J^{\dot{A}} J^{\dot{B}}
$$

The key function and the curvature are

$$
\begin{aligned}
& W=W(\phi, w, t) \\
& C^{(3)}=-2 \mu_{0} \phi^{3}, \quad C^{(1)}=6 \tau \phi^{7} \mu_{0} W_{t}, \quad \mu_{0}=\mathrm{const} \neq 0 \\
& C_{\dot{A} \dot{B} \dot{C} \dot{D}}=-\phi^{3} J_{(\dot{A}} J_{\dot{B}} C_{\dot{C}} D_{\dot{D})}, \quad C_{(\dot{C}} D_{\dot{D})}=\frac{6 \mu_{0}}{\tau^{2}} K_{\dot{C}} K_{\dot{D}}-W_{\phi \phi \phi \phi} J_{\dot{C}} J_{\dot{D}}
\end{aligned}
$$

The metric reads

$$
\begin{aligned}
d s^{2}=2 \phi^{-2} & \left\{\tau^{-1}(d \eta \underset{s}{\otimes} d w-d \phi \underset{s}{\otimes} d t)+\left(\frac{\mu_{0}}{\tau^{2}} \phi^{3}+\frac{\Lambda}{6 \tau^{2}}\right) d t \underset{s}{\otimes} d t\right. \\
& \left.+\left(2 W_{\phi}-\phi W_{\phi \phi}\right) d w \underset{s}{\otimes} d w\right\}
\end{aligned}
$$

After inserting the key function (5.3) into the hyperheavenly equation we get

$$
\left(\mu_{0} \phi^{3}+\frac{\Lambda}{6}\right) W_{\phi \phi}-3 \mu_{0} \phi^{2} W_{\phi}+3 \mu_{0} \phi W+\tau W_{t \phi}=0
$$

In order to maintain the type [II] $\otimes[\mathrm{III}]$ we have to assume $W_{t} \neq 0$ and $W_{\phi \phi \phi \phi} \neq 0$. The general solution of the Eq. (5.6) is not known. Its reduction to canonical form is realized by the transformation

$$
s:=\frac{\mu_{0}}{\tau} t, \quad z:=\frac{\mu_{0}}{\tau} t-\int \frac{d \phi}{\phi^{3}+\frac{\Lambda}{6 \mu_{0}}}
$$

Considering the key function $W$ as a function of the variables $(z, s, w)$ we obtain the equation

$$
W_{z s}-6 \phi^{2} W_{z}-3 \phi\left(\phi^{3}+\frac{\Lambda}{6 \mu_{0}}\right) W=0
$$

Multiplying Eq. (5.8) by $\phi\left(\phi^{3}+\frac{\Lambda}{6 \mu_{0}}\right)^{-2}$ one can bring it to the form

$$
\partial_{s}\left[\phi\left(\phi^{3}+\frac{\Lambda}{6 \mu_{0}}\right)^{-2} W_{z}\right]-\partial_{z}\left[\left(\phi^{3}+\frac{\Lambda}{6 \mu_{0}}\right)^{-1} W\right]=0
$$

From (5.9) we infer the existence of the potential $\Sigma=\Sigma(z, s, w)$ such that

$$
\begin{aligned}
& \Sigma_{z}=\phi\left(\phi^{3}+\frac{\Lambda}{6 \mu_{0}}\right)^{-2} W_{z} \\
& \Sigma_{s}=\left(\phi^{3}+\frac{\Lambda}{6 \mu_{0}}\right)^{-1} W
\end{aligned}
$$


From the (5.10b) one can calculate $W$

$$
W=\left(\phi^{3}+\frac{\Lambda}{6 \mu_{0}}\right) \Sigma_{s}
$$

Inserting it into (5.10a) we arrive to the equation

$$
\Sigma_{z s}=3 \phi^{2} \Sigma_{s}+\phi^{-1}\left(\phi^{3}+\frac{\Lambda}{6 \mu_{0}}\right) \Sigma_{z}
$$

Of course, $\phi$ has to be considered as a function of coordinates $s$ and $z, \phi=\phi(z, s)$, according to (5.7). The integral in (5.7) can be calculated in all subcases, leading to the condition $z=z(s, \phi)$. Unfortunately, the inverse function $\phi=\phi(z, s)$ is an elementary function only if $\Lambda=0$. However, if $\Lambda=0$ more efficient transformation can be proposed.

\subsubsection{Special case $\Lambda=0$}

In what follows we assume $\Lambda=0$ and we introduce the transformation $(\phi, \eta, w, t) \rightarrow$ $(x, y, u, v)$ :

$\phi^{2}=\left(-\frac{1}{\mu_{0}}\right)^{\frac{2}{3}} \frac{1}{4 x}, \quad \frac{t}{\tau}=\left(-\frac{1}{\mu_{0}}\right)^{\frac{1}{3}}(x+i y), \quad \frac{w}{\tau}=-\frac{1}{4}\left(-\frac{1}{\mu_{0}}\right)^{\frac{2}{3}} u, \quad \eta=v$

In terms of the coordinates $(x, y, u, v)$ the metric (5.5) has the form

$$
d s^{2}=-2 x d u \underset{s}{\otimes}(d v+M d u)+x^{-\frac{1}{2}}(d x \underset{s}{\otimes} d x+d y \underset{s}{\otimes} d y)
$$

where

$$
M:=-\frac{\tau^{2}}{4}\left(-\frac{1}{\mu_{0}}\right)^{\frac{2}{3}}\left(2 W_{\phi}-\phi W_{\phi \phi}\right) \equiv 2 \tau^{2}\left(-\frac{1}{\mu_{0}}\right)^{\frac{1}{3}}\left(\partial_{x}+i \partial_{y}\right)\left(x^{\frac{5}{2}}\left(\partial_{x}+i \partial_{y}\right) W\right)
$$

Obviously, $M$ is a function of the variables $(x, y, u)$. The hyperheavenly Eq. (5.6) takes the form

$$
4 x^{2}\left(W_{x x}+W_{y y}\right)+12 x\left(W_{x}+i W_{y}\right)+3 W=0
$$

differentiating Eq. (5.16) twice by $\partial_{x}+i \partial_{y}$ and using definition of $M$, after some algebraic work we obtain

$$
x M_{x x}+x M_{y y}+M_{x}=0
$$

Equation (5.17) is equivalent to the Euler-Poisson-Darboux equation (EPD equation) and its solutions have been discussed in literature [35]. The form of the metric (5.14) and the Eq. (5.17) are especially useful in obtaining the Lorentzian slices (see Sect. 6). 


\subsection{Spaces of the type $[\mathrm{D}]^{e} \otimes[\mathrm{D}]^{e}$}

From the previous subsection one can easily obtain the general metric for the type $[\mathrm{D}]^{e} \otimes[\mathrm{D}]^{e}$ with $\Lambda$. Self-dual type [D] we get after setting $C^{(1)}=0 \Rightarrow W_{t}=$ $0 \Rightarrow W=W(\phi, w)$. General solution of the hyperheavenly Eq. (5.6) reads $W=$ $\mu_{0} f_{1}(w) \phi^{3}+f_{2}(w) \phi-(\Lambda / 3) f_{1}(w)$ and it automatically causes anti-self-dual type being of the type [D]. Moreover, it can be proved that arbitrary functions $f_{1}$ and $f_{2}$ can be gauged to zero without any loss of generality. [We do not prove that fact here, but it can be easily done by using the results from [29] together with some straightforward calculations]. Finally it brings us to conclusion, that in the hyperheavenly spaces of the type $[D] \otimes[D]$ admitting null Killing vector the key function $W$ can be gauged to zero. [The inverse implication is also true; putting $W=0$ in expanding hyperheavenly equation and all curvature formulas we easily get, that such hyperheavenly space automatically becomes of the type $[\mathrm{D}] \otimes[\mathrm{D}]$ with the metric (5.18)]. The metric

$$
d s^{2}=(\phi \tau)^{-2}\left\{2 \tau(d \eta \underset{s}{\otimes} d w-d \phi \underset{s}{\otimes} d t)+2\left(\mu_{0} \phi^{3}+\frac{\Lambda}{6}\right) d t \underset{s}{\otimes} d t\right\}
$$

admits, together with null isometric Killing vector $\partial_{\eta}$, three other isometric Killing vectors $\partial_{w}, \partial_{t}$ and $w \partial_{w}-\eta \partial_{\eta}$ and - if $\Lambda=0$ - one homothetic Killing vector $\frac{2}{3} \chi_{0}\left(2 t \partial_{t}-\right.$ $\left.\phi \partial_{\phi}+\eta \partial_{\eta}\right)$.

If $\Lambda=0$ the metric (5.18) can be easily transformed to coordinate system ( $x, y, u, v)$ defined by (5.13). According to (5.15) function $M=0$ and the metric reads

$$
d s^{2}=-2 x d u \underset{s}{\otimes} d v+x^{-\frac{1}{2}}(d x \underset{s}{\otimes} d x+d y \underset{s}{\otimes} d y)
$$

\subsection{Spaces of the type $[\mathrm{III}]^{e} \otimes[\mathrm{III}]^{e}$ and $[\mathrm{N},-]^{e} \otimes[\mathrm{N},-]^{e}$}

The next possible metrics generated by null Killing vector with both self-dual and antiself-dual congruences of expanding null strings, are metrics of the type $[\mathrm{III}]^{e} \otimes[\mathrm{III}]^{e}$, $[\mathrm{N}]^{e} \otimes[\mathrm{N}]^{e}$ and $[\mathrm{N}]^{e} \otimes[-]^{e}$ or $[-]^{e} \otimes[\mathrm{N}]^{e}$. All of them have been found in [29]. Here we have the Killing vector

$$
K=\frac{\partial}{\partial \eta}
$$

and

$$
l_{A B}=\frac{\Lambda \phi}{3 \tau} \delta_{(A}^{2} \delta_{B)}^{2}, \quad l^{\dot{A} \dot{B}}=-\frac{2}{\tau} \phi^{-1} J^{\dot{A}} J^{\dot{B}}
$$

The key function and the curvature

$$
\begin{aligned}
& W=\alpha_{0} \eta \phi^{3}+f(z, w)-\frac{3}{7 \Lambda} \tau^{2} \alpha_{0}^{2} \phi^{7}+g_{t} \phi^{2}-\frac{\Lambda}{3 \tau} g \phi, \quad z:=\phi-\frac{\Lambda t}{6 \tau} \\
& C^{(2)}=-2 \tau \alpha_{0} \Lambda \phi^{5}, \quad C^{(1)}=-4 \tau^{2} \phi^{7}\left(g_{t t t}+\alpha_{0}^{2} \Lambda \phi^{3}\right) \\
& C_{\dot{A} \dot{B} \dot{C} \dot{D}}=\phi^{3} J_{(\dot{A}} J_{\dot{B}} J_{\dot{C}} L_{\dot{D})}
\end{aligned}
$$




$$
L_{\dot{A}}:=\left(f_{z z z z}-\frac{360}{\Lambda} \tau^{2} \alpha_{0}^{2} \phi^{3}\right) J_{\dot{A}}-24 \alpha_{0} K_{\dot{A}}
$$

where $f=f(z, w)$ and $g=g(w, t)$ are arbitrary functions of their variables. The metric reads

$$
\begin{aligned}
d s^{2}= & (\phi \tau)^{-2}\left\{2 \tau(d \eta \underset{s}{\otimes} d w-d \phi \underset{s}{\otimes} d t)+\frac{\Lambda}{3} d t \underset{s}{\otimes} d t-8 \tau^{2} \alpha_{0} \phi^{3} d w \underset{s}{\otimes} d t\right. \\
& \left.+2\left(-\tau^{2} f_{z z} \phi+2 \tau^{2} f_{z}+\frac{12}{\Lambda} \tau^{4} \alpha_{0}^{2} \phi^{6}+2 \tau^{2} g_{t} \phi-\frac{2}{3} \tau \Lambda g\right) d w \underset{s}{\otimes} d w\right\}
\end{aligned}
$$

In all formulas $\Lambda \neq 0$. Particular types are characterized by

- type $[\mathrm{III}]^{e} \otimes[\mathrm{III}]^{e}: \alpha_{0} \neq 0, \alpha_{0}$ can be re-gauged to 1 without any loss of generality

- type $[\mathrm{N}]^{e} \otimes[\mathrm{N}]^{e}: \alpha_{0}=0, g_{t t t} \neq 0, f_{z z z z} \neq 0$

- type $[\mathrm{N}]^{e} \otimes[-]^{e}: \alpha_{0}=0, g_{t t t} \neq 0, f_{z z z z}=0$ (one can set $f=0$ without any loss of generality)

- type $[-]^{e} \otimes[\mathrm{N}]^{e}: \alpha_{0}=0, f_{z z z z} \neq 0, g_{t t t}=0$ (one can set $g=0$ without any loss of generality)

Of course, spaces of the types $[\mathrm{N}]^{e} \otimes[-]^{e}$ and $[-]^{e} \otimes[\mathrm{N}]^{e}$ have the same geometry of null strings, since both self-dual and anti-self-dual congruences of null strings are expanding. The case $[-]^{e} \otimes[\mathrm{N}]^{e}$ has been considered with details in [30]. It describes the general heavenly metric with $\Lambda$ admitting null Killing vector. In real case this solution has the signature (++- $)$ ) and it is general metric of the 4-dimensional global Osserman space with non-zero curvature scalar admitting the null Killing vector.

\subsection{Spaces of the type $[\mathrm{III}, \mathrm{N},-]^{n} \otimes[\mathrm{N},-]^{e}$}

In this case we deal with the hyperheavenly spaces of types $[\mathrm{III}, \mathrm{N}]^{n} \otimes[\mathrm{N}]^{e}$ with nonexpanding congruence of self-dual null strings defined by the null Killing vector; the congruence of anti-self-dual null strings is still expanding. The respective metrics have been discussed in [28]. The Killing vector has the form

$$
K=q^{\mathrm{i}} \frac{\partial}{\partial p^{\mathrm{i}}}
$$

Then

$$
l_{A B}=-\delta_{(A}^{2} \delta_{B)}^{2}, \quad l^{\dot{A} \dot{B}}=0
$$

The key function and the curvature read

$$
\begin{aligned}
& \theta=p^{\dot{2}} S\left(q^{\dot{2}}, q^{\mathrm{i}} p^{\dot{2}} p^{\dot{2}}\right)-\frac{1}{12} \frac{p^{\dot{2}}}{q^{\dot{1}}}\left(F_{0} p^{\dot{2}}-p^{\mathrm{i}}\right)\left(F_{0} p^{\dot{2}}-3 p^{\dot{1}}\right)+\frac{1}{2} N p^{\dot{2}} p^{\dot{2}} \\
& C^{(2)}=F_{0} \frac{1}{q^{\dot{1}} q^{\dot{1}}}, \quad C^{(1)}=-2 \frac{\partial}{\partial q^{\dot{1}}}\left(\frac{N}{2 q^{\dot{1}}}-\frac{\partial N}{\partial q^{\dot{1}}}\right)-\frac{2 F_{0} p^{\dot{1}}}{\left(q^{\dot{1}}\right)^{3}}-\frac{F_{0}^{2} p^{\dot{2}}}{\left(q^{\dot{1}}\right)^{3}}
\end{aligned}
$$




$$
C_{\dot{A} \dot{B} \dot{C} \dot{D}}=\frac{1}{2} \dot{C}^{(1)} \delta_{\dot{A}}^{\dot{2}} \delta_{\dot{B}}^{\dot{2}} \delta_{\dot{C}}^{\dot{2}} \delta_{\dot{D}}^{\dot{2}}, \quad \dot{C}^{(1)}=2\left(p^{\dot{2}} S\left(q^{\dot{2}}, q^{\dot{1}} p^{\dot{2}} p^{\dot{2}}\right)\right)_{p^{\dot{2}} p^{\dot{2}} p^{2} p^{2}}
$$

where $N=N\left(q^{\dot{M}}\right)$ and $S=S\left(q^{\dot{2}}, q^{\dot{1}} p^{\dot{2}} p^{\dot{2}}\right)$ are arbitrary functions and $F_{0}$ is a constant. The metric takes the form

$$
\begin{aligned}
d s^{2}= & -2 d p^{\dot{A}} \underset{s}{\otimes} d q_{\dot{A}}-2\left(\left(p^{\dot{2}} S\right)_{p^{\dot{2}} p^{\dot{2}}}-\frac{F_{0}^{2}}{2} \frac{p^{\dot{2}}}{q^{\dot{1}}}+N\right) d q_{\mathrm{i}} \underset{s}{\otimes} d q_{\mathrm{i}} \\
& +\frac{p^{\dot{2}}}{q^{\dot{1}}} d q_{\dot{2}} \underset{s}{\otimes} d q_{\dot{2}}+2\left(2 F_{0} \frac{p^{\dot{2}}}{q^{\dot{1}}}-\frac{p^{\dot{1}}}{q^{\dot{1}}}\right) d q_{\mathrm{i}} \underset{s}{\otimes} d q_{\dot{2}}
\end{aligned}
$$

$\Lambda$ necessarily must be zero and the hyperheavenly metrics are characterized by

- type $[\mathrm{III}]^{n} \otimes[\mathrm{N}]^{e}: F_{0} \neq 0, F_{0}$ can be re-gauged to 1 without any loss of generality, $\left(p^{2} S\right)_{p^{2}} p^{2} p^{2} p^{2} \neq 0$

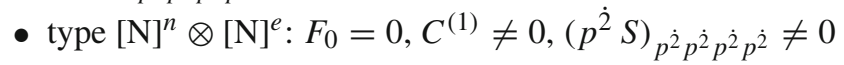

The heavenly reductions of the metric obtained above are especially interesting, because they provide two different null string geometries. One can get the metrics of the type $[\mathrm{III}, \mathrm{N}]^{n} \otimes[-]^{e}$ or $[-]^{n} \otimes[\mathrm{N}]^{e}$.

In order to obtain the heavenly metrics of the types [III,N $]^{n} \otimes[-]^{e}$ one must set $S\left(q^{\dot{2}}, q^{\dot{1}} p^{\dot{2}} p^{\dot{2}}\right)=f\left(q^{\dot{2}}\right) q^{\dot{1}} p^{\dot{2}} p^{\dot{2}}$ where $f$ is an arbitrary function of the variable $q^{\dot{2}}$. (It seems, that this is too strong condition and it is enough to set $\left(p^{\dot{2}} S\right){ }_{p^{2}} p^{2} p^{\dot{2}} p^{2}=0$, but there is unused gauge freedom, which allows to simplify the function $S$ ). Heavenly metrics of the type $[-]^{n} \otimes[\mathrm{N}]^{e}$ can be obtained by setting $F_{0}=0=N$ (once again condition $N=0$ is stronger then necessary $C^{(1)}=0$, but $N=0$ can be obtained by using gauge freedom). Finally

- type $[\mathrm{III}]^{n} \otimes[-]^{e}: F_{0} \neq 0, F_{0}$ can be re-gauged to 1 without any loss of generality, $S=f\left(q^{\dot{2}}\right) q^{\dot{1}} p^{\dot{2}} p^{\dot{2}}$

- type $[\mathrm{N}]^{n} \otimes[-]^{e}: F_{0}=0, C^{(1)} \neq 0, S=f\left(q^{\dot{2}}\right) q^{\dot{1}} p^{\dot{2}} p^{\dot{2}}$

- type $[-]^{n} \otimes[\mathrm{N}]^{e}: F_{0}=0, N=0,\left(p^{\dot{2}} S\right)_{p^{2} p^{2} p^{2} p^{2}} \neq 0$

\subsection{Spaces of the type $[\mathrm{N},-]^{n} \otimes[\mathrm{N},-]^{n}$}

The last case is characterized by both self-dual and anti-self-dual congruences of null strings being nonexpanding. The only possible types are $[\mathrm{N},-]^{n} \otimes[\mathrm{N},-]^{n}$. These metrics have been found in [28]. The Killing vector takes the form

$$
K=\frac{\partial}{\partial p^{\mathrm{i}}}
$$

with spinors $l_{A B}$ and $l_{\dot{A} \dot{B}}$ being

$$
l_{A B}=0, \quad l^{\dot{A} \dot{B}}=0
$$


The key function and the curvature are

$$
\begin{aligned}
& \theta=\frac{1}{2} N\left(q^{\dot{M}}\right) p^{\dot{2}} p^{\dot{2}}+A\left(p^{\dot{2}}, q^{\dot{2}}\right) \\
& C^{(1)}=2 \frac{\partial^{2} N}{\partial q^{\dot{1}} \partial q^{\dot{1}}}, \quad \dot{C}^{(1)}=2 A_{p^{2} p^{2} p^{\dot{2}} p^{\dot{2}}}
\end{aligned}
$$

where $N=N\left(q^{\dot{M}}\right)$ and $A=A\left(p^{\dot{2}}, q^{\dot{2}}\right)$ are arbitrary functions. The metric reads

$$
d s^{2}=-2 d p^{\dot{A}} \otimes_{s} d q_{\dot{A}}-2\left(A_{p^{2} p^{2}}+N\right) d q_{\mathrm{i}} \underset{s}{\otimes} d q_{\mathrm{i}}
$$

There are two different heavenly degenerations but they lead to the equivalent heavens. To get the heavenly space of the type $[-]^{n} \otimes[\mathrm{N}]^{n}$ it is enough to set $N_{q^{\mathrm{i}}} q^{\mathrm{i}}=0$, then by using gauge freedom one can gauge $N$ away. The heavenly space of the type $[\mathrm{N}]^{n} \otimes[-]^{n}$ can be obtained by setting the function $A$ as a third-order polynomial in $p^{2}$ (in fact, taking into considerations the remaining gauge freedom, it is enough to set $\left.A=f\left(q^{\dot{2}}\right) p^{\dot{2}} p^{\dot{2}} p^{\dot{2}}\right)$. Gathering, we arrive at the cases

- type $[\mathrm{N}]^{n} \otimes[\mathrm{N}]^{n}: N_{q^{\mathrm{i}} q^{\mathrm{i}}} \neq 0, A_{p^{2} p^{2} p^{2} p^{2}} \neq 0$

- type $[-]^{n} \otimes[\mathrm{N}]^{n}: N=0, A_{p^{2} p^{2} p^{2} p^{2}} \neq 0$

- type $[\mathrm{N}]^{n} \otimes[-]^{n}: N_{q^{\mathrm{i}} q^{\mathrm{i}}} \neq 0, A=f\left(q^{\dot{2}}\right) p^{\dot{2}} p^{\dot{2}} p^{\dot{2}}$

\section{Real slices}

\subsection{Real slices with neutral signature $(++--)$}

The metrics presented in Sects. 4 and 5 are holomorphic. It is an easy matter to carry over all the results to the case of real spaces of the signature $(++--)$. To this end, instead of the holomorphic objects (spinors, null strings, tetrads, coordinates, etc.) we simply deal with the real smooth objects. Real spaces of the neutral signature play an important role in Walker and Osserman geometry [36-38]. Recently, it has been recognized that the hyperheavenly formalism allows to obtain transparent results in Walker and Osserman geometry. For example, a new class of metrics admitting selfdual and anti-self-dual, parallely propagated null strings (two-sided Walker spaces) has been found in [25]. These spaces have a natural generalization when only one of the families of null strings is parallely propagated (sesqui-Walker spaces). Such spaces have been defined and investigated in [38]. Probably the most distinguished success of the hyperheavenly methods in Osserman geometry was finding all algebraically degenerate metrics of the globally Osserman space which do not have the Walker property, i.e. they do not admit any parallely propagated null strings [26].

Some of the metrics presented in Sects. 4 and 5 are examples of the Walker or Osserman spaces admitting the null Killing vector. These metrics are gathered in the table: 
sesqui-Walker two-sided Walker globally Osserman
(4.6), (4.9), (5.28) of the types [III,N,- $]^{n} \otimes[\mathrm{N}]^{e}$

(4.14), (5.28) of the types [III,N,- $]^{n} \otimes[-]^{e},(5.33)$

(4.9), (4.14), (5.23) of the type $[\mathrm{N}]^{e} \otimes[-]^{e}$ or $[-]^{e} \otimes[\mathrm{N}]^{e}$, (5.28) of the type $[\mathrm{III}, \mathrm{N},-]^{n} \otimes[-]^{e}$ and $[-]^{n} \otimes[\mathrm{N},-]^{e}$,

(5.33) of the type $[\mathrm{N}]^{n} \otimes[-]^{n}$ or $[-]^{n} \otimes[\mathrm{N}]^{n}$

Among globally Osserman spaces the most interesting is the one with metric (5.23) of the type $[\mathrm{N}]^{e} \otimes[-]^{e}$ or $[-]^{e} \otimes[\mathrm{N}]^{e}$. It does not have any parallely propagated null strings (because of $\Lambda \neq 0$ ). Consequently it is the most general globally Osserman but not Walker space equipped with a null isometric Killing vector.

\subsection{Real Lorentzian slices}

Of course, the most interesting from the physical point of view are Lorentzian slices. There are still no general techniques of obtaining such slices, except some notes on their properties [23]. However, in some special cases they can be obtained quite easily.

It is well known, that there are only two subcases of the Einstein spaces with Lorentzian signature and null Killing vector (compare [39]). One of them is pp-wave solution. The real metric of the pp-wave solution can be obtained from the complex metric (5.33) of the type $[\mathrm{N}]^{n} \otimes[\mathrm{N}]^{n}$. Detailed discussion of this case can be found in [28]. We only mention, that in this particular case it is enough to consider the necessary condition of existing Lorentzian slice, namely $C_{\dot{A} \dot{B} \dot{C} \dot{D}}=\bar{C}_{A B C D}$, which gives now $\dot{C}^{(1)}=\bar{C}^{(1)}$.

Except the pp-wave solution, null Killing vector is admitted by the Lorentzian, Einstein spaces of the type [II] and [D]. It means, that desired Lorentzian slice is hidden in the hyperheavenly metric (5.5) with the key function (5.3) and with curvature given by (5.4). Unfortunatelly, in this case the conditions $C_{\dot{A} \dot{B} \dot{C} \dot{D}}=\bar{C}_{A B C D}$ are not straightforward and technique which succeeded in pp-wave case, failed.

However, one can consider the (complex) transformation (5.13) which brings the metric to the form (5.14). The metric (5.14) depends on one (complex) function $M=$ $M(x, y, u)$ which satisfies the Eq. (5.17). Treating now the coordinates $(x, y, u, v)$ as real coordinates and the function $M$ as a real smooth function we find the metric (5.14) automatically becomes real and has the Lorentzian signature. The vacuum Einstein equations have been reduced to the Eq. (5.17). Exactly the same form of the metric with the same equation describing real vacuum Lorentzian types [II] and [D] admitting a null isometric Killing vector can be found in [39]. Summing up, the metric (5.14) with real coordinates is another example of Lorentzian slice of the complex space.

Why does this technique succeed? The first reason is, probably, the explicit use of the imaginary unit in transformation (5.13). It plays no role if we consider (5.13) as complex transformation and the coordinates $(x, y, u, v)$ as complex. But if $(x, y, u, v)$ are real, this step changes automatically the signature of the metric making (5.14) Lorentzian. The second reason is that the metric (5.14) does not depend directly on the key function $W$ but on the function $M$. The relation between this two functions 
is given by (5.15) and it contains the imaginary unit. However, differentiating twice the hyperheavenly Eq. (5.16) one can bring it to the form (5.17) which is free of imaginary unit. Finally, we are left with the real metric and the real equation. It is enough to accomplish the construction of Lorentzian spaces of the type [II] and [D] admitting null Killing vector.

It is worth to note that this construction works only in the vacuum case. If cosmological constant $\Lambda \neq 0$ we have not been able to find the Lorentzian slices.

We conclude also that no Einstein spaces with null homothetic symmetries admit Lorentzian slices.

\section{Concluding remarks}

In this paper the null Killing vectors (isometric and homothetic) in complex spacetime have been considered. The relation between the existence of null Killing vector and geometry of null strings has been studied in Sect. 2. Because of the existence of null strings the most natural apparatus in investigating null Killing vectors appeared to be the one provided by the theory of hyperheavenly and heavenly spaces. After short summary of the structure of hyperheavenly spaces (Sect. 3), we have been able to present all possible metrics admitting null Killing vector. Only two of them i.e.

- the metric (4.6) of the type $[\mathrm{N}]^{e} \otimes[\mathrm{III}]^{n}$ with null homothetic Killing vector

- the metric (5.5) of the types $[\mathrm{II}]^{e} \otimes[\mathrm{III}]^{e}$ with $\Lambda \neq 0$ and with null isometric Killing vector

have not been solved completely. In (4.6) the functions $F=F(x, w, t)$ and $\gamma=$ $\gamma(w, t)$ satisfy the Eq. (4.5). No solution with $F_{x x x} \neq 0$ and $\gamma_{t} \neq 0$ have been found. However, the geometry of this space is so interesting and the type $[\mathrm{N}]^{e} \otimes[\mathrm{III}]^{n}$ so rare, that we are going to study the Eq. (4.5) with details in future. As for the metric (5.5) we have been able to solve the case $[\mathrm{D}]^{e} \otimes[\mathrm{D}]^{e}$ and reduce the type $[\mathrm{II}]^{e} \otimes[\mathrm{II}]^{e}$ with $\Lambda=0$ to the Euler-Poisson-Darboux equation which solutions are known. The type $[\mathrm{II}] \otimes[\mathrm{II}]$ with $\Lambda \neq 0$ has been reduced to the Eq. (5.12), but this reduction has obvious disadvantages. Like in the previous case, we will deal with this equation soon.

The transparent results are the metrics (4.9) and (4.14) which constitute all heavens with null homothetic symmetry. These cases have been considered in [40] but without giving any explicit form of the metric. We were able to integrate the problem completely.

Perhaps, the most interesting from the physical point of view is searching for examples of real Lorentzian slices of the complex metrics. The first such an example has been presented in [28]. Here we have been able to find the Lorentzian slices of the types $[\mathrm{II}]^{e} \otimes[\mathrm{II}]^{e}$ and $[\mathrm{D}]^{e} \otimes[\mathrm{D}]^{e}$ with $\Lambda=0$. They are given by the metric (5.14) which depends on one function $M$ of three variables satisfying the Eq. (5.17). Such a metric has been presented earlier (see [41], or in a concise form [39]).

Both these examples gave some valuable hints about obtaining Lorentzian slices. In the first of them the condition $C_{\dot{A} \dot{B} \dot{C} \dot{D}}=\bar{C}_{A B C D}$ has been successfully used, in the second one a reasonable using of imaginary unit appears to be essential. Nonetheless, the case with nonzero cosmological constant is still unsolved. Taking into considerations the optical properties of the congruences of null geodesics defined by the null 
isometric Killing vector (2.30a) - (2.30c) we conclude that all such slices must belong to the Kundt class ([39], xxxi). The vacuum Einstein field equations with cosmological constant for the Kundt class have been gathered in [39] but no explicit solution has been presented there. The vacuum types [II] and [D] with cosmological constant admitting null isometric Killing vector via Lorentzian slices of complex types $[\mathrm{II}]^{e} \otimes[\mathrm{II}]^{e}$ and $[\mathrm{D}]^{e} \otimes[\mathrm{D}]^{e}$ become our main issue to be considered in future.

However, interesting observation can be immediately made. The generic complex spacetimes admitting null isometric Killing vector which have the Lorentzian slices are equipped with congruence of null strings which have the same properties. Indeed, pp-wave solution can be obtained from the complex spacetime of the type $[\mathrm{N}]^{n} \otimes[\mathrm{N}]^{n}$ where both self-dual and anti-self-dual congruences of null strings are nonexpanding. In the opposite, Lorentzian types [II] and [D] have been obtained from the complex spacetimes of the types $[\mathrm{III}]^{e} \otimes[\mathrm{II}]^{e}$ and $[\mathrm{D}]^{e} \otimes[\mathrm{D}]^{e}$ in which both congruences of null strings are expanding. Maybe it is a general rule and Lorentzian slices can be obtained only from the complex spacetimes equipped with both expanding or both nonexpanding null strings?

We hope, that further investigations on the structure of complex spacetimes allow us to find some effective and more general techniques of obtaining real Lorentzian slices.

Open Access This article is distributed under the terms of the Creative Commons Attribution License which permits any use, distribution, and reproduction in any medium, provided the original author(s) and the source are credited.

\section{References}

1. Penrose, R.: A spinor approach to general relativity. Ann. Phys. 10, 171 (1960)

2. Newman, E.T., Penrose, R.: An approach to gravitational radiation by a method of spin coefficients. J. Math. Phys. 3, 566 (1962)

3. Debney, G.C., Kerr, R.P., Schild, A.: Solutions of the Einstein and Einstein-Maxwell equations. J. Math. Phys. 10, 1842 (1969)

4. Newman, E.T., Janis, A.I.: Note on the Kerr spinning-particle metric. J. Math. Phys. 6, 915 (1965)

5. Demiański, M., Newman, E.T.: Combined Kerr-Nut solution of the Einstein field equations. Bull. Acad. Pol. Sci. 14, 653 (1966)

6. Trautman, A.: Analytic solutions of Lorentz-invariant linear equations. Proc. Roy. Soc. (London) A270-A326 (1962)

7. Newman, E. T.: Heaven and its properties riddle of gravitation symposium syracuse. (1975)

8. Plebański, J.F.: Some solutions of complex Einstein equations. J. Math. Phys. 16, 2395 (1975)

9. Plebański, J.F., Przanowski, M., Formañski, S.: Linear superposition of two type-N nonlinear gravitons. Phys. Lett. A 246, 25 (1998)

10. Plebański, J.F., Robinson, I.: Left—degenerate vacuum metrics. Phys. Rev. Lett. 37, 493 (1976)

11. Flaherty, E.J.: Hermitian and Kählerian Geometry in Relativity. Springer, Berlin (1976)

12. Finley III, J.D., Plebański, J.F.: The intrinsic spinorial structure of hyperheavens. J. Math. Phys. 17, 2207 (1976)

13. Plebański, J. F., Robinson, I.: The complex vacuum metric with minimally degenerated conformal curvature. In: Esposito, F. P., Witten, L. (eds.) Asymptotic Structure of Space-Time, pp. 361-406. Plenum Publishing Corporation, New York (1977)

14. Boyer, C. P., Finley III, J. D., Plebański, J. F.: Complex general relativity, $\mathcal{H}$ and $\mathcal{H} \mathcal{H}$ spaces - a survey to one approach. In: Held, A. (ed.) General Relativity and Gravitation. Einstein Memorial Volume, vol. 2, pp. 241-281. Plenum, New York (1980) 
15. Plebański, J.F., Hacyan, S.: Some properties of Killing spinors. J. Math. Phys. 14, 2204 (1976)

16. Finley III, J.D., Plebański, J.F.: The clasification of all $\mathcal{H}$ spaces admitting a Killing vector. J. Math. Phys. 20, 1938 (1979)

17. Plebański, J.F., Finley, J.D.: Killing vectors in nonexpanding HH spaces. J. Math. Phys. 19, 760 (1978)

18. Sonnleitner, S.A., Finley III, J.D.: The form of Killing vectors in expanding $\mathcal{H} \mathcal{H}$ spaces. J. Math. Phys. 23(1), 116 (1982)

19. García, D. A., Plebański, J. F.: Seven parametric type-D solutions of Einstein-Maxwell equations in the basic left—degenerate representation Il Nuovo Cimento Serie 11. 40, B224 (1977)

20. Robinson, D.C.: Some real and complex solutions of Einstein's equations. Gen. Relativ. Gravit. 19, 7 (1987)

21. Hickman, M.S., McIntosh, C.B.: Complex relativity and real solutions. III. Real type-N solutions from complex N $\otimes$ N ones. Gen. Relativ. Gravit. 18, 107 (1986)

22. Robinson, D.C.: Holomorphic 4-metrics and Lorentzian structures. Gen. Relativ. Gravit. 34(8), 1173 (2002)

23. Rózga, K.: Real slices of complex space-time in general relativity. Rep. Math. Phys. 11, 197 (1977)

24. Finley III, J. D.: Toward real—valued $\mathcal{H} \mathcal{H}$ spaces: twisting type N. In: Rindler, W., Trautman, A. (eds.) Gravitation and Geometry, pp. 130-158. Bibliopolis, Naples (1987)

25. Chudecki, A., Przanowski, M.: From hyperheavenly spaces to Walker and Osserman spaces: I. Class. Quantum Grav. 25, 145010 (2008)

26. Chudecki, A., Przanowski, M.: From hyperheavenly spaces to Walker and Osserman spaces: II. Class. Quantum Grav. 25, 235019 (2008)

27. Chudecki, A.: Conformal Killing vectors in Nonexpanding $\mathcal{H} \mathcal{H}$-spaces with $\Lambda$. Class. Quantum Grav. 27, 205004 (2010)

28. Chudecki, A.: Classification of the Killing vectors in nonexpanding $\mathcal{H} \mathcal{H}$-spaces with $\Lambda$. Class. Quantum Grav. 29, 135010 (2012)

29. Chudecki, A.: Homothetic killing vectors in expanding $\mathcal{H} \mathcal{H}$-spaces with $\Lambda$. Int. J. Geom. Methods Mod. Phys. 10(1), 1250077 (2013)

30. Chudecki, A., Przanowski, M.: Killing symmetries in $\mathcal{H}$-spaces with $\Lambda$. J. Math. Phys. 54, 102503 (2013)

31. Plebański, J. F., Przanowski, M.: Null tetrad, spinor and helicity formalism for all 4-dimensional Riemannian manifolds. I. Null tetrads and spinors. Acta Phys. Polon. B 19(10), 805-825 (1988)

32. Penrose, R., Rindler, W.: Spinors and Space-Time, p. 458. Cambridge University Press, Cambridge (1984)

33. Plebański, J.F., Hacyan, S.: Null geodesic surfaces and Goldberg-Sachs theorem in complex Riemannian spaces. J. Math. Phys. 16, 2403 (1975)

34. Plebański, J.F., Rózga, K.: The optics of null strings. J. Math. Phys. 25, 1930 (1984)

35. Koshlyakov, N.S., Smirnov, M.M., Gliner, E.B.: Differential Equations of Mathematical Physics. North Holland Publishing Company, Amsterdam (1964)

36. Law, P.R., Matsushita, Y.: A spinor approach to Walker geometry. Commun. Math. Phys. 282(3), 577-623 (2008)

37. Law, P.R., Matsushita, Y.: Algebraically special real alpha geometries. J. Geom. Phys. 61, 2064-2080 (2011)

38. Law, P.R., Matsushita, Y.: Real AlphaBeta-geometries and Walker geometry. J. Geom. Phys. 65, 35-44 (2013)

39. Stephani, H., Kramer, D., MacCallum, M.A.H., Hoenselaers, C., Herlt, E.: Exact Solutions to Einstein's Field Equations, 2nd edn. Cambridge University Press, Cambridge (2003)

40. Dunajski, M., West, S.: Anti-self-dual conformal structures with null killing vectors from projective structures. Commun. Math. Phys. 272, 85-118 (2007)

41. Dautcourt, G.: Relativistic theories of gravitation. In: Infeld, L. (ed.) Gravitationsfelder Mit Isotropem Killingvektor, p. 300. Pergamon Press, Oxford (1964) 